\title{
Inhibitor of apoptosis-stimulating protein of p53 inhibits ferroptosis and alleviates intestinal ischemia/reperfusion-induced acute lung injury
}

\author{
Yingchuan $\mathrm{Li}^{1} \cdot$ Yongmei Cao ${ }^{1} \cdot$ Jian Xiao ${ }^{2} \cdot$ Jiawei Shang ${ }^{1} \cdot$ Qin $\operatorname{Tan}^{1} \cdot$ Feng Ping ${ }^{1} \cdot$ Weifeng Huang ${ }^{1} \cdot$ Fang Wu $^{1}$. \\ Haijun Zhang ${ }^{3} \cdot$ Xiaoping Zhang ${ }^{3,4}$
}

Received: 1 September 2019 / Revised: 2 March 2020 / Accepted: 6 March 2020 / Published online: 18 March 2020

(c) The Author(s), under exclusive licence to ADMC Associazione Differenziamento e Morte Cellulare 2020

\begin{abstract}
Acute lung injury (ALI) is a life-threatening disorder with high rates of morbidity and mortality. Reactive oxygen species and epithelial apoptosis are involved in the pathogenesis of acute lung injury. Ferroptosis, an iron-dependent non-apoptotic form of cell death, mediates its effects in part by promoting the accumulation of reactive oxygen species. The inhibition of ferroptosis decreases clinical symptoms in experimental models of ischemia/reperfusion-induced renal failure and heart injury. This study investigated the roles of inhibitor of apoptosis-stimulating protein of p53 (iASPP) and Nrf2 in ferroptosis and their potential therapeutic effects in intestinal ischemia/reperfusion-induced acute lung injury. Intestinal ischemia/ reperfusion-induced ALI was induced in wild-type and $\mathrm{Nrf}_{2}^{-l-}$ mice. The mice were treated with erastin followed by liproxstatin-1. Ferroptosis-related factors in mice with ischemia/reperfusion-induced acute lung injury or in mouse lung epithelial-2 cells with hypoxia/regeneration (HR)-induced ALI were measured by western blotting, real-time PCR, and immunofluorescence. Ferroptosis contributed to intestinal ischemia/reperfusion-induced ALI in vivo. iASPP inhibited ferroptosis and alleviated intestinal ischemia/reperfusion-induced acute lung injury, and iASPP-mediated protection against ischemia/reperfusion-induced ALI was dependent on Nrf2 signaling. HR-induced acute lung injury enhanced ferroptosis in vitro in mouse lung epithelial-2 cells, and ferroptosis was modulated after the enhancement of intestinal ischemia/ reperfusion in $\mathrm{Nrf}^{-/-}$mice. iASPP mediated its protective effects against acute lung injury through the Nrf2/HIF-1/TF signaling pathway. Ferroptosis contributes to intestinal ischemia/reperfusion-induced ALI, and iASPP treatment inhibits ferroptosis in part via Nrf2. These findings indicate the therapeutic potential of iASPP for treating ischemia/reperfusioninduced ALI.
\end{abstract}

These authors contributed equally: Yingchuan Li, Yongmei Cao, Jian Xiao

Edited by M. Piacentini

Supplementary information The online version of this article (https:// doi.org/10.1038/s41418-020-0528-x) contains supplementary material, which is available to authorized users.

Yingchuan Li

yingchuan.li@sjtu.edu.cn

$\triangle$ Haijun Zhang zhanghaijun@tongji.edu.cn

$\triangle$ Xiaoping Zhang zxpsibs@163.com

1 Department of Critical Care Medicine, Shanghai Jiaotong University Affiliated Sixth People's Hospital, Shanghai, China

\section{Introduction}

Acute lung injury (ALI) is a critical life-threatening disorder with very high rates of morbidity and mortality among patients with numerous medical conditions $[1,2]$. The pathogenesis of ALI is mediated by activated platelets that promote innate immune reactions, including pulmonary

2 Department of Cardiothoracic Surgery, Changzheng Hospital, Second Military Medical University, Shanghai, China

3 The Institute of Intervention Vessel, Tongji University School of Medicine, Shanghai, China

4 Shanghai Center of Thyroid Diseases, Tongji University School of Medicine, Shanghai, China 
neutrophil recruitment, and the production and secretion of proteases and toxic mediators [3, 4]. The clinical features of ALI include the tissue infiltration of inflammatory cells, pulmonary edema, and arterial hypoxemia, which damage the vascular endothelium and alveolar epithelium, thus diminishing lung function [5-7].

Interestingly, injuries to tissues distant from the lung, such as abdominal injury, infection, and surgery, that induce intestinal ischemia/reperfusion (I/R) can also cause ALI [2, 8]. The generation of reactive oxygen species (ROS) and high levels of epithelial apoptosis are critical in the pathogenesis of this type of ALI, which is known as intestinal I/R-induced ALI [8]. Because no effective treatments are currently available for ALI, the development of potential treatments to target these factors has been suggested [9]. In addition, iron mobilization and decompartmentalization have a pathogenic role in animal models and human cases of ALI [10].

Ferroptosis is an iron-dependent, p53-mediated, nonapoptotic form of cell death, which mediates its effects in part by promoting the accumulation of lethal lipid ROS [11]. It is involved in various human diseases, and its inhibition is effective in diminishing clinical symptoms in experimental models of $\mathrm{I} / \mathrm{R}$-induced renal failure and heart injury [12-14]. Ferroptosis has also been reported to promote tumor cell death, and small molecules that induce ferroptosis, such as erastin, have potential in cancer treatments [15]. Mediators of islet oxidative stress, including glutathione peroxidase-4 (GPX4), have been identified as inhibitors of ferroptosis, and mechanisms that affect GPX4 function can influence islet function and viability [16].

p53, a tumor suppressor, is stimulated by apoptosisstimulating protein of p53 and consequently induces apoptosis and ferroptosis in response to DNA damage [15]. Interestingly, inhibitor of apoptosis-stimulating protein of p53 (iASPP) inhibits p53-induced apoptosis and facilitates tumor growth, and the overexpression of iASPP induces chemoresistance in human cancer cells [15]. Of relevance to ALI, iASPP exerts substantial anti-ROS activity independently of p53 in the cytoplasm, and promotes the accumulation and nuclear translocation of nuclear factor (erythroidderived 2)-like 2 (Nrf2) [15], a transcription factor that confers cellular protection against oxidative stress induced by various types of cell death, including ferroptosis, apoptosis, and autophagy [17]. Of note, Nrf2 activation in the nucleus has been reported to protect against numerous airway disorders/diseases, including ALI [18]. In addition, nuclear Nrf2 interacts with the transcriptional coactivator small v-maf avian musculoaponeurotic fibrosarcoma oncogene homolog (Maf) proteins such as MafG and then activates transcription of quinone oxidoreductase-1 (NQO-1), heme oxygenase-1 (HO-1), and ferritin heavy chain 1 (FTH1). Knockdown of NQO-1, HO-1, and FTH1 by RNAi in HCC cells accelerates ferroptosis [17].
Collectively, these studies indicate the therapeutic potential of iASPP and Nrf2 and the targeting of ferroptosis for treating ALI. Currently, there is a need to develop novel treatments to decrease patient mortality and morbidity due to ALI. However, the roles of ferroptosis, iASPP, and Nrf2 in ALI remain unclear. Therefore, this study examined the roles of iASPP and Nrf2 in ferroptosis, as well as their potential therapeutic effects in treating intestinal I/Rinduced lung injury in vivo and in vitro.

\section{Materials and methods}

\section{Animal model of intestinal I/R-induced ALI}

All experiments were performed in accordance with the established guidelines, under approval from the Animal Care and Usage Committee of Shanghai Ninth People's Hospital (China). All animal procedures were performed in accordance with institutional guidelines. 8-week-old Nrf2knockout $\left(\mathrm{Nrf}^{-\mathrm{C}^{-}}\right)$and wild-type (WT) littermate male mice on a C57BL/6J background (provided by the RIKEN Bio-Resource Center through the National Bio-Resource Project of MEXT, Japan) were used for in vivo experiments. Animals were fasted for $24 \mathrm{~h}$ before experiments and given free access to water. Intestinal ischemia was induced by clamping of the superior mesenteric artery after the intraperitoneal injection of $50 \mathrm{mg} / \mathrm{kg}$ of sodium pentobarbital. After $90 \mathrm{~min}$, the intestine was reperfused for the times indicated. Sham control mice underwent the same procedure without vascular occlusion. During the entire process, three mice died and were not included in the experiment.

To determine the effect of ferroptosis on I/R-induced ALI, we treated mice with erastin $(10 \mu \mathrm{M}$; MedChem Express) daily for 20 days, then with either liproxstatin-1 $(1 \mu \mathrm{M}$; Sigma-Aldrich) or no treatment daily for another 20 days. Erastin was dissolved in 5\% dimethylsulfoxide + corn oil (C8267, Sigma-Aldrich, St. Louis, MO, USA) at $37^{\circ} \mathrm{C}$ in a water bath with gentle shaking.

In some cases, mice were also treated with small interfering (si) RNAs against iASPP (by Gene Pharma Corporation, Shanghai, China) to knockdown expression of iASPP (see "Cell culture and treatment" for sequences).

\section{Histopathological analysis}

Lung samples were fixed in 10\% formalin and then embedded in paraffin. Tissue blocks were cut into 5- $\mu \mathrm{m}$ slices, stained with hematoxylin and eosin (H\&E), and analyzed under a light microscope. Lung injury was analyzed by an experienced investigator blinded to the level of induced injury (absent, mild, moderate, or severe; score $0-3$ ) on the basis of the presence of exudates, hyperemia 
and congestion, neutrophilic infiltrates, intra-alveolar hemorrhage and debris, and cellular hyperplasia. In brief, edema, atelectasis, necrosis, alveolar and interstitial inflammation, and hemorrhage, and hyaline membrane formation were each scored on a scale of 0-4: 0 , no injury; 1, 25\% injury; 2, 50\% injury; $3,75 \%$ injury; and $4,100 \%$ injury. Each injury was scored in ten randomly selected fields (200x) from each slide.

\section{Immunofluorescence staining}

Lung tissue sections were deparaffinized, permeabilized with $0.1 \%$ Triton $\mathrm{X}-100$, and blocked with $5 \%$ bovine serum albumin before incubation with anti-Nrf2 and anti-iASPP antibodies overnight at $4{ }^{\circ} \mathrm{C}$. Sections were then washed five times in phosphate buffered saline (PBS) and incubated with green-fluorescent Alexa Fluor 488 donkey anti-rabbit IgG (Invitrogen, Grand Island, NY, USA) or red-fluorescent Alexa Fluor 594 rabbit anti-mouse IgG (Invitrogen).

After washing in PBS, mounting, and staining with $4^{\prime}, 6-$ diamidino-2-phenylindole (DAPI), slides were observed, and images were captured with a fluorescence microscope (Olympus FluoView ${ }^{\mathrm{TM}}$ FV1000, Tokyo, Japan).

Mouse lung epithelial (MLE)-2 cells were fixed in $2 \%$ paraformaldehyde for $10 \mathrm{~min}$ at room temperature, washed with PBS, permeabilized for 10 min with $0.5 \%$ Triton X-100, and blocked for $1 \mathrm{~h}$ in blocking buffer (1\% bovine serum albumin, $10 \%$ goat serum, and $0.1 \%$ Triton X-100 in PBS).

Alexa Fluor-conjugated secondary antibodies were incubated for $1 \mathrm{~h}$ at room temperature. DNA was visualized with DAPI $(0.5 \mu \mathrm{g} / \mathrm{ml})$. Images were acquired with a fluorescence microscope (Olympus FluoView ${ }^{\mathrm{TM}}$ FV1000) for confocal imaging.

\section{Enzyme-linked immunosorbent assay (ELISA) of cytokines}

Mice were euthanized, and lungs were lavaged with $1 \mathrm{ml}$ (Hank's Balanced Salt Solution, HBSS) (Invitrogen, Carlsbad, CA, USA) through the tracheal cannula to obtain bronchoalveolar lavage fluid (BALF). Interleukin (IL)-1 $\beta$, IL6 , and tumor necrosis factor (TNF)- $\alpha$ concentrations were measured with ELISA kits (Westang Biotech, Shanghai, China) according to the manufacturer's instructions.

\section{Cell culture and treatment}

MLE-2 cells were purchased from the ATCC (Manassas, VA, USA) and cultured in RPMI 1640 medium (Invitrogen) supplemented with $5 \%$ heat-inactivated fetal bovine serum, L-glutamine, penicillin, and streptomycin at $37{ }^{\circ} \mathrm{C}$ and $5 \%$ $\mathrm{CO}_{2}$ in humidified air. iASPP siRNA (si-iASPP), iASPP vector (iASPP), and Nrf2 siRNAs (si-Nrf2) were designed and chemically synthesized by Gene Pharma Corporation (Shanghai, China) and transfected into the cells with Lipofectamine RNAi Max (Invitrogen). The sequences of the si-iASPP and si-Nrf2 oligonucleotides were as follows: siiASPP: 5'-GGCTGACAGAAACAAGCAT-3'; and si-Nrf2: 5'-CCGGCATTTCACTAAACACAA-3'.

To induce hypoxia, we placed cells in a hypoxic chamber with $5 \% \mathrm{CO}_{2}, 94 \% \mathrm{~N}_{2}$, and $1 \% \mathrm{O}_{2}$ at $37^{\circ} \mathrm{C}$ for $2 \mathrm{~h}$, and this was followed by reoxygenation for $0,3,6$, or $12 \mathrm{~h}$.

\section{Western blot analysis}

Western blotting was used to analyze protein expression. In brief, after extraction, proteins in cell lysates were resolved by sodium dodecyl sulfate polyacrylamide gel electrophoresis, transferred to a polyvinylidene difluoride membrane, and incubated with a primary antibody. After incubation with peroxidase-conjugated secondary antibodies, the signals were visualized by enhanced chemiluminescence (Pierce, Rockford, IL, USA) according to the manufacturer's instructions. The primary antibodies used were anti-iASPP (1:200, Sigma), anti-Nrf2 (1:1000; Abcam, Cambridge, MA, USA), anti-HIF-1 $\alpha \quad(1 ; 1000$, Novus Biologicals, LLC, Littleton, CO, USA), anti-GPX4 (1;1000, Abcam), anti-HO-1 (1:1500, Santa Cruz Biotechnology, Dallas, TX, USA), anti-transferrin (TF) $(1 ; 500$, Abcam); anti-Lamin B (1:1500, Abcam); anti-ACSL4 (1:10000, Abcam); anti-FTH1 (1:1000, Abcam); antiNQO-1 (1:1000, Abcam), and anti-GAPDH (1:1000, Abcam). All experiments were performed in triplicate. The quantitative results of western blot analysis were determined in Image $\mathbf{J}$ software (US National Institutes of Health, Bethesda, MD, USA).

\section{$\mathrm{Fe}^{2+}$, glutathione (GSH), and lipid peroxidation assays}

The relative iron concentration in cell lysates was assessed with an Iron Assay Kit (ab83366; Abcam). The relative concentration of malondialdehyde (MDA) in cell lysates was assessed with a Lipid Peroxidation Assay Kit (ab118970; Abcam). The relative GSH concentration in cell or tissue lysates was assessed with a Glutathione Assay Kit (CS0260; Sigma, USA). All kits were used according to the manufacturers' instructions. NQO-1 and HO-1 activity was measured with commercially available ELISA kits (Westang Biotechnology, Shanghai, China).

\section{RT-PCR analysis}

Total RNA was extracted with an RNeasy Mini kit (Qiagen, Hamburg, Germany), and $2 \mu \mathrm{g}$ of RNA was reverse transcribed to complementary DNA (40 ng) template. The 
amplification cycling reactions (40 cycles) were as follows: $5 \mathrm{~s}$ at $95^{\circ} \mathrm{C}$ and $30 \mathrm{~s}$ at $60^{\circ} \mathrm{C}$. Relative quantification values of the target genes were standardized according to the comparative threshold cycle $\left(2^{-\Delta \Delta C T}\right)$. Primer sequences and PCR settings were as follows: iASPP: forward, $5^{\prime}-\mathrm{GA}$ AATCACTGGGGACAGGAA-3', reverse, 5'-CCCAGGA ATATCCAGTGGTG-3'; Nrf2: forward, 5'-CACATCCAG TCAGAAACCAGTGG- ${ }^{\prime}$, reverse, 5'-GGAATGTCTGC GCCAAAAGCTG-3'; ferritin heavy chain (FTH1): forward, 5'-TGAAGCTGCAGAACCAACGAGG-3', reverse, 5'-GCACACTCCATTGCATTCAGCC-3'; and NQO-1: forward, 5'-CCTGCCATTCTGAAAGGCTGGT, reverse, 5'-GTGGTGATGGAAAGCACTGCCT-3'.

\section{Statistical analysis}

All results are expressed as the means \pm standard deviation. Statistical analysis was performed with Student's $t$ test. One-way analysis of variance was used for multiple comparisons. $P$ values $<0.05$ were considered to indicate a statistically significant difference.

\section{Results}

\section{Intestinal I/R-induced ALI enhances ferroptosis in vivo}

Figure 1a-c shows the induction of I/R-induced ALI and the pulmonary function of ALI model mice. As the duration of $\mathrm{I} / \mathrm{R}$ increased, $\mathrm{PaO}_{2}$ significantly decreased, and the percentage water weight of the lungs and BALF protein content significantly increased, as compared with the results in sham mice. H\&E staining of lungs showed that, with increasing I/R duration, edema, atelectasis, necrosis, alveolar and interstitial inflammation, hemorrhage, and hyaline membrane formation were aggravated, as compared with the observations in sham mice (Fig. 1d). Masson's staining to assess pulmonary fibrosis with increasing $\mathrm{I} / \mathrm{R}$ duration showed more collagen deposits than those in sham mice (Fig. 1d). The relative values of GSH, MDA, and $\mathrm{Fe}^{2+}$, oxidative stress markers associated with I/R-induced ALI were assessed. The percentage GSH significantly decreased, and the percentage MDA and $\mathrm{Fe}^{2+}$ levels significantly increased with increasing $\mathrm{I} / \mathrm{R}$ duration, as compared with the results in sham mice (Fig. 1e-g). GPX4 is a phospholipid hydroperoxidase that protects cells against membrane lipid peroxidation. The protein and mRNA levels of the ferroptosis markers FTH1 and GPX4 significantly decreased, and TF (an iron transporter that prevents the formation of ROS) [19] significantly increased with increasing $\mathrm{I} / \mathrm{R}$ duration, as compared with the results in sham mice (Fig. 1h-j).
Erastin-induced ferroptosis promotes intestinal I/Rinduced $A L I$ in vivo

Erastin activates a rapid, oxidative, non-apoptotic cell death process and appears to function as activator of ferroptosis $[11,20,21]$. Liproxstatin-1 has been shown to suppress ferroptosis in cells and in a preclinical model of I/R-induced hepatic damage [22]. The mechanism through which liproxstatin-1 prevents cell death has been ascribed to its ability to inhibit lipid peroxidation directly by trapping chain-carrying radicals [23]. Erastin treatment of I/R mice significantly increased the percentage MDA, $\mathrm{Fe}^{2+}$ levels, and protein/mRNA levels of TF and significantly decreased the percentage GSH and protein/mRNA levels of FTH1 and GPX4, but these effects were reversed by liproxstatin-1 treatment (Fig. 2a-f). H\&E and Masson's staining of lung tissues showed that erastin aggravated edema, atelectasis, necrosis, alveolar and interstitial inflammation, and pulmonary fibrosis in $\mathrm{I} / \mathrm{R}$ mice compared with sham mice, but these effects were reversed in $I / R+$ erastin mice treated with liproxstatin-1 (Fig. 2g). Cytokine levels (IL-1 $\beta$, IL-6, and TNF- $\alpha$ ) were significantly higher in BALF from I/R + erastin mice than sham mice, and these effects was reversed in $\mathrm{I} / \mathrm{R}+$ erastin mice treated with liproxstatin-1 (Fig. S1A-C).

\section{iASPP knockdown promotes intestinal I/R-induced $A L I$, and iASPP overexpression inhibits intestinal I/R- induced $A L I$ in WT mice}

To address the role of iASPP in intestinal I/R-induced ALI, we detected the expression of iASPP in lung tissues and found that the protein and mRNA expression increased in lung tissues with increasing I/R duration, as compared with the results in sham mice (Fig. 3a). iASPP protein decreased in the nucleus and increased in the cytoplasm $60 \mathrm{~min}$ after I/ $\mathrm{R}$, as compared with the results in sham mice (Fig. 3b-d). Levels of IL- $1 \beta$, IL- 6 , and TNF- $\alpha$ were significantly higher in BALF from $\mathrm{I} / \mathrm{R}$ mice than from sham mice, and increased further when mice were treated with adenovirus encoding si-iASPP but not si-Ctrl (Fig. S2A-C). These effects were reversed when iASPP was overexpressed in I/R mice (Fig. S2D-F). Histological analysis showed that si-iASPP aggravated edema, atelectasis, necrosis, alveolar and interstitial inflammation, and pulmonary fibrosis, but these effects were reversed by iASPP overexpression (Fig. 3e, f). These findings indicated that the absence of iASPP increased susceptibility to lung tissue injury.

\section{iASPP overexpression inhibits ferroptosis, and iASPP knockdown promotes ferroptosis in vivo}

To characterize the role of iASPP in ferroptosis, we assessed the expression of several gene involved in iron 
A

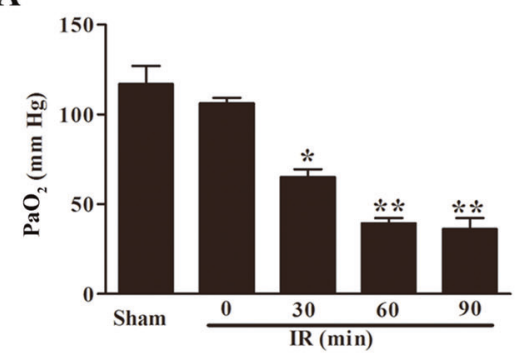

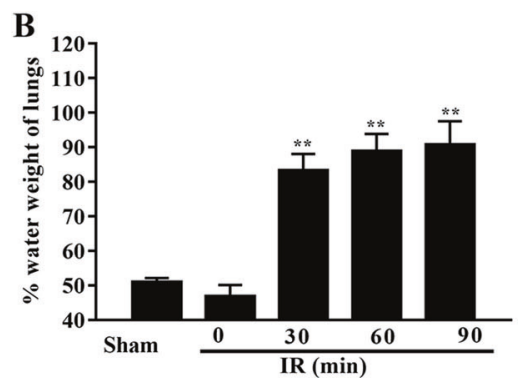

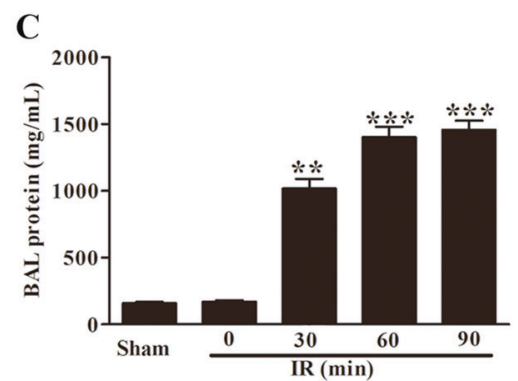

D
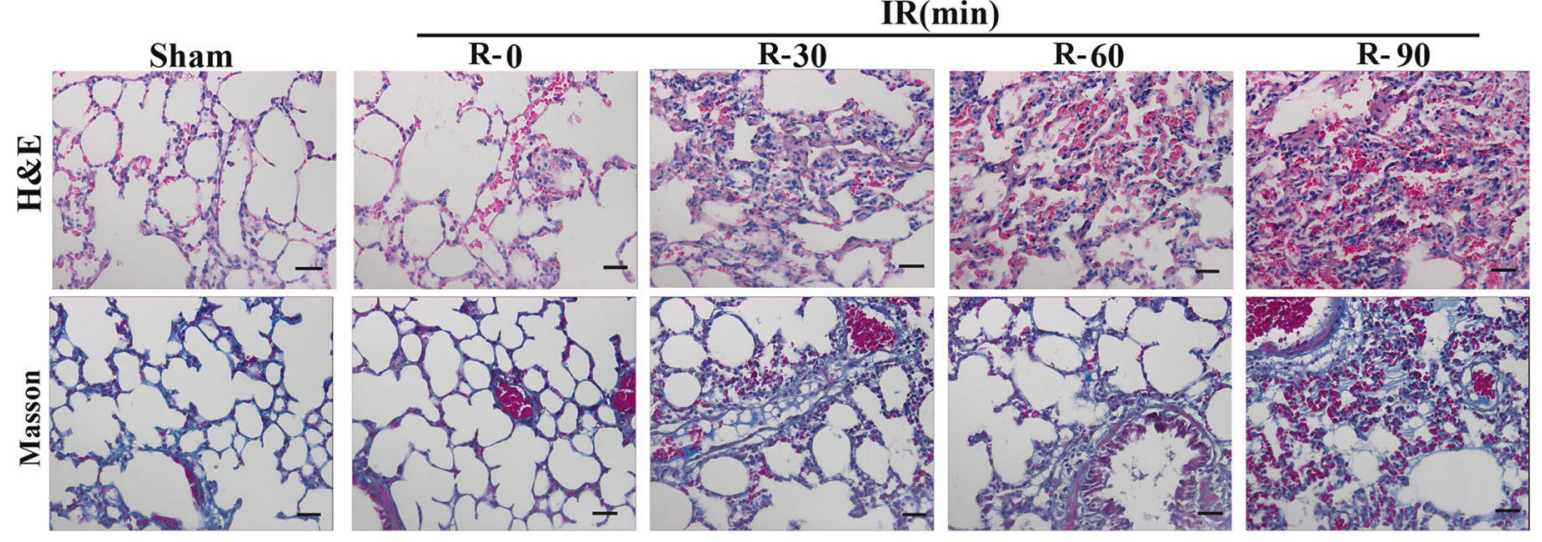

E

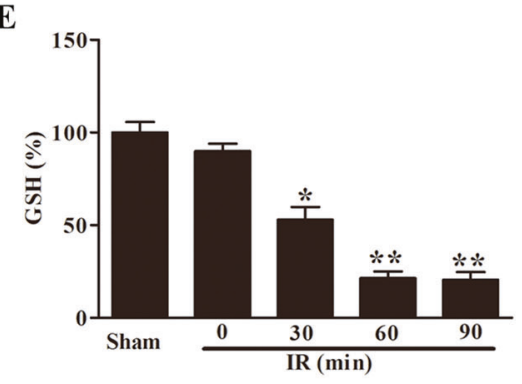

F

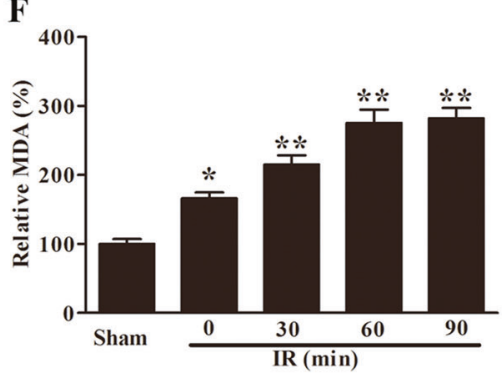

G

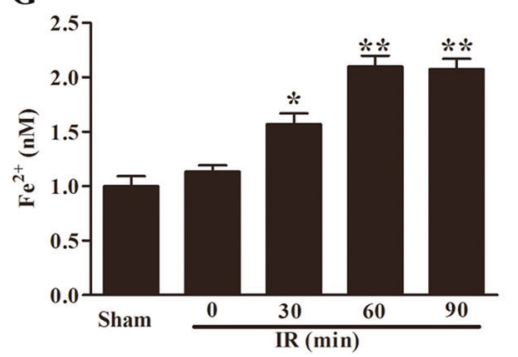

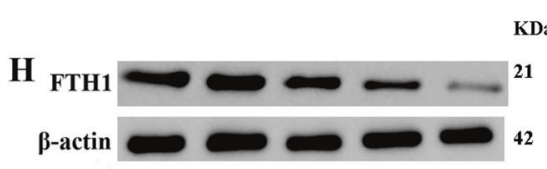

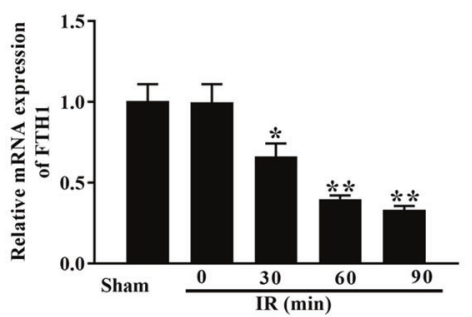

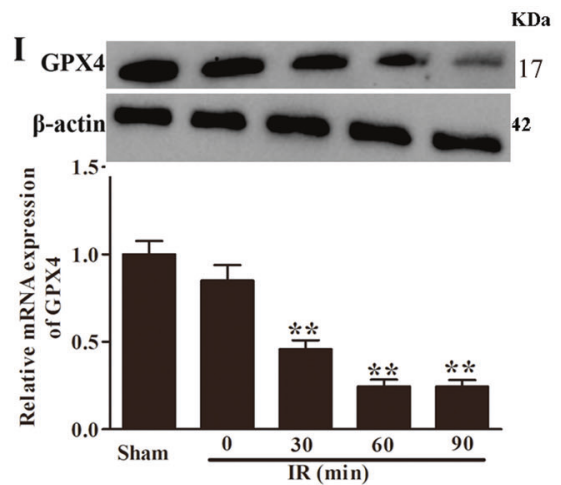

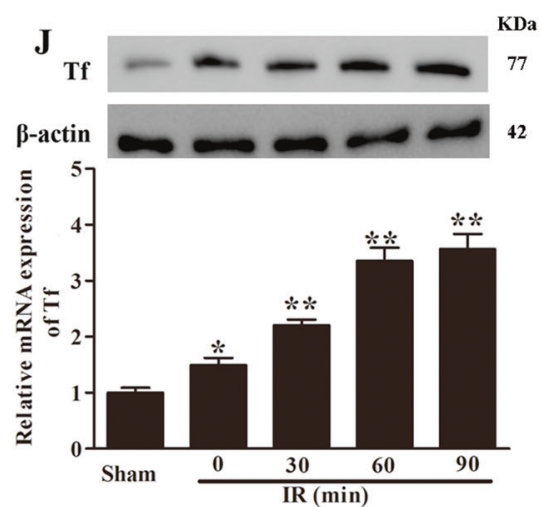

Fig. 1 Intestine I/R-induced ALI enhances ferroptosis in vivo. C57BL/6 mice were subjected to $60 \mathrm{~min}$ of intestinal ischemia followed by $0,30,60$, or 90 min of reperfusion as indicated. Sham mice were included as controls. a Arterial blood $\mathrm{PaO}_{2}$ was detected and lung water content was analyzed (b). c The protein content of BALF was measured. Results are representative of three experiments $(n=6$

metabolism and lipid peroxidation in mice transfected with adenovirus encoding iASPP or control sequences. The protein and mRNA expression of FTH1 and GPX4 were per group). d Representative H\&E- and Masson trichrome-stained lung sections. Morphology was examined using light microscopy. Scar bar $=20 \mu \mathrm{m}$. The relative values of GSH (e) and MDA (f) and the $\mathrm{Fe}^{2+}$ concentration $(\mathbf{g})$ were measured. $\mathbf{h}-\mathbf{j}$ The ferroptosis-related proteins FTH1, GPX4, and TF were analyzed by RT-PCR and western blot analysis. $* p<0.05, * * p<0.01$.

significantly decreased, however, the expression of TF and ACSL4 were significantly increased, $60 \mathrm{~min}$ after $\mathrm{I} / \mathrm{R}$ compared with sham mice, and were decreased further in 
A

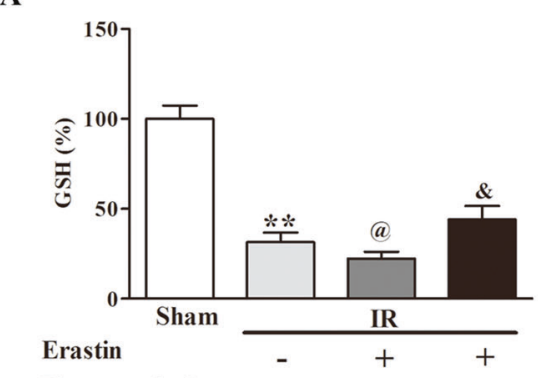

Liproxstatin-1

D
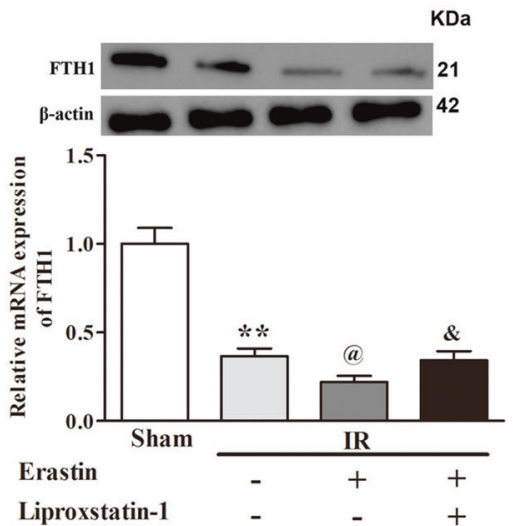

B

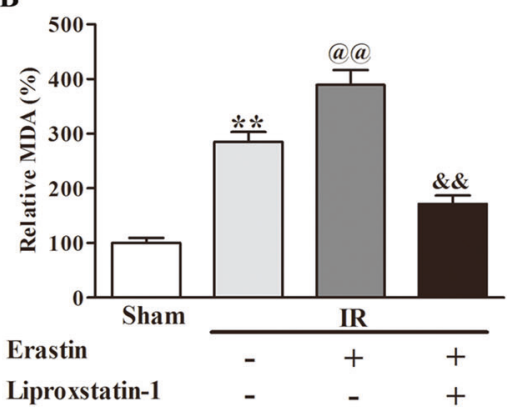

E
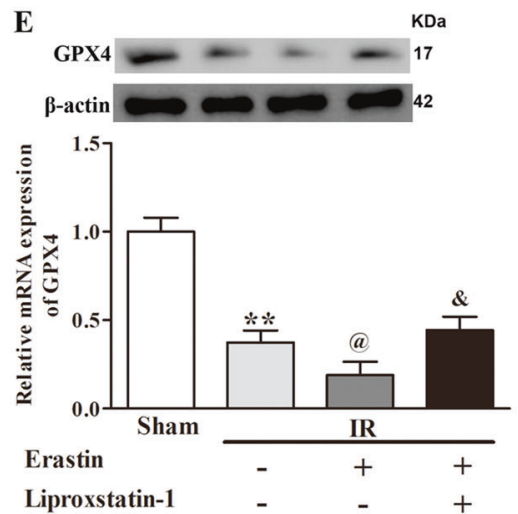

C

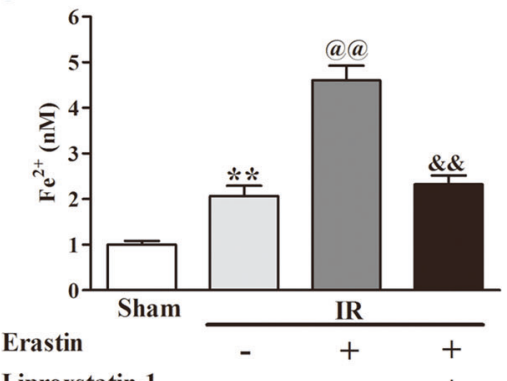

Liproxstatin-1 $\quad-\quad$ - $\quad+$

$\mathbf{F}$

KDa
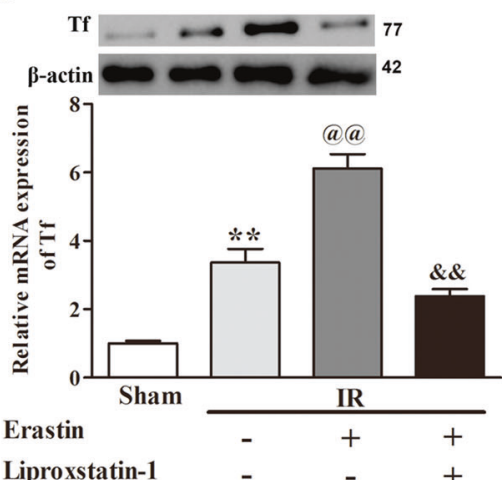

G

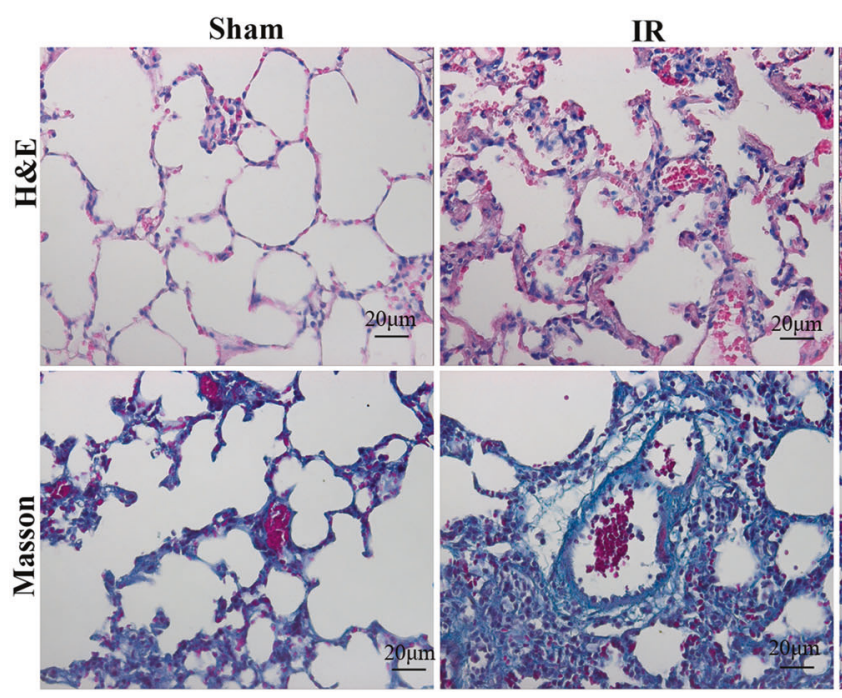

Fig. 2 Erastin-induced ferroptosis promotes I/R-induced ALI injury in vivo. C57BL/6 mice were subjected to $60 \mathrm{~min}$ of intestinal ischemia followed $60 \mathrm{~min}$ of reperfusion $(\mathrm{I} / \mathrm{R})$ as indicated, and then erastin $(10 \mu \mathrm{M})$ was tail-injected intravenously for 20 days, followed by treatment with liproxstatin-1 $(1 \mu \mathrm{M})$ for 20 days. Sham mice were included as controls. The relative values of GSH (a) and MDA (b) and

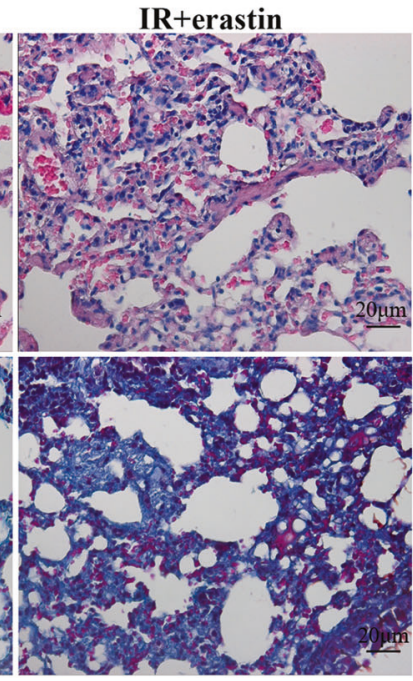

IR+erastin+Liproxstatin-1

the $\mathrm{Fe}^{2+}$ concentration (c) were measured. $\mathbf{d}-\mathbf{f}$ The ferroptosis-related proteins FTH1, GPX4, and TF were analyzed by RT-PCR and western blot analysis. g Representative H\&E- and Masson trichrome-stained lung sections. Morphology was examined using light microscopy. Scar bar $=20 \mu \mathrm{m} . * * p<0.01$ vs. Sham; ${ }^{@} p<0.05$ vs. IR; ${ }^{\circledR} p<0.01$ vs. IR; ${ }^{\&} p<0.05$ vs. IR + Erastin; ${ }^{\& \&} p<0.01$ vs. IR + Erastin.

mice transfected with adenovirus encoding iASPP compared with the vector control (Fig. 4a-e), but decreased in mice receiving adenovirus encoding iASPP compared with si-Ctrl (Fig. 4f-j). The data above demonstrating that the iASPP expression is crucial for the extent of ferroptosis induced by ALI in vivo.

\section{iASPP-mediated intestinal I/R-induced ALI is dependent on Nrf2 signaling in vivo}

The above research provide evidence that iASPP play critical roles in intestinal I/R-induced ALI, but the link between them is still not clear. We next investigate whether 

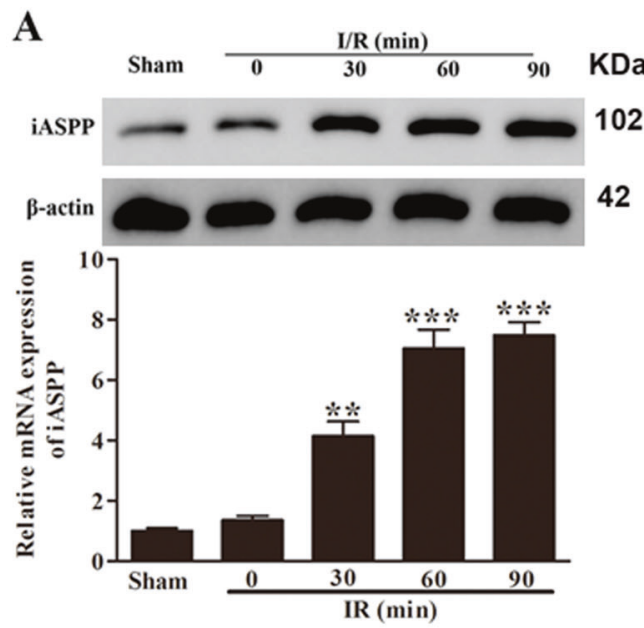

$\mathbf{E}$

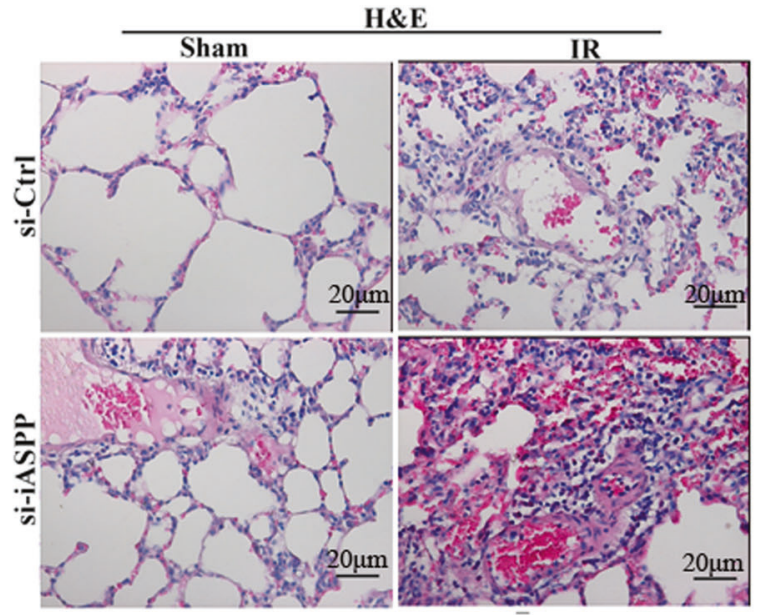

$\mathbf{F}$

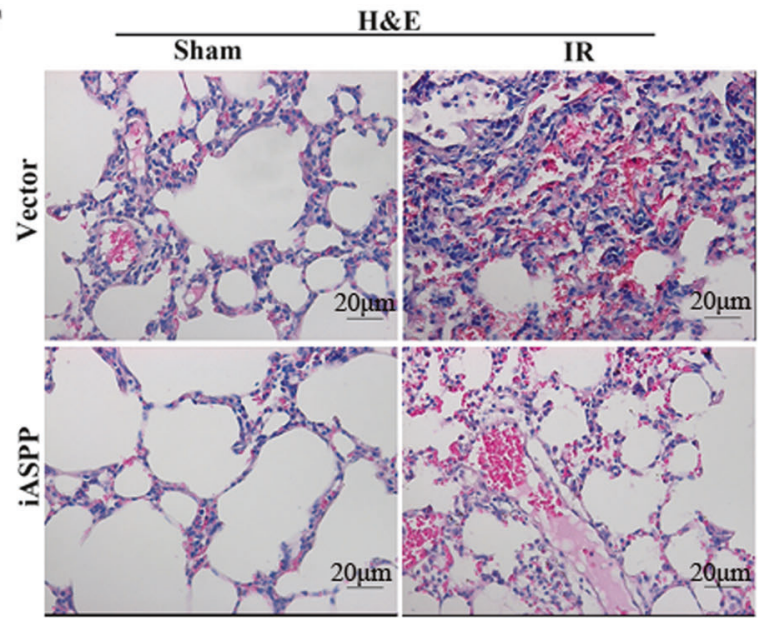

Fig. 3 The effect of iASPP knockdown or overexpression on intestinal I/R-induced ALI in WT mice. a The relative mRNA and protein expressions of iASPP were detected in lung tissues in the sham group and I/R groups by RT-PCR and western blotting. b, $\mathbf{c}$ The protein expression of iASPP in the nucleus and cytoplasm following I/R (60 min/60 min) was measured by western blotting. d Representative photographs showing iASPP staining in lung tissues


IR

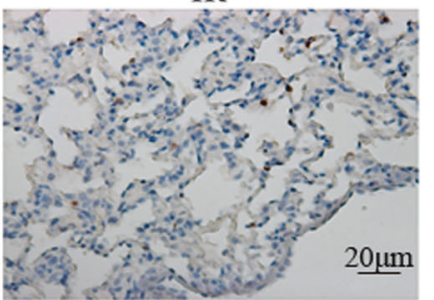

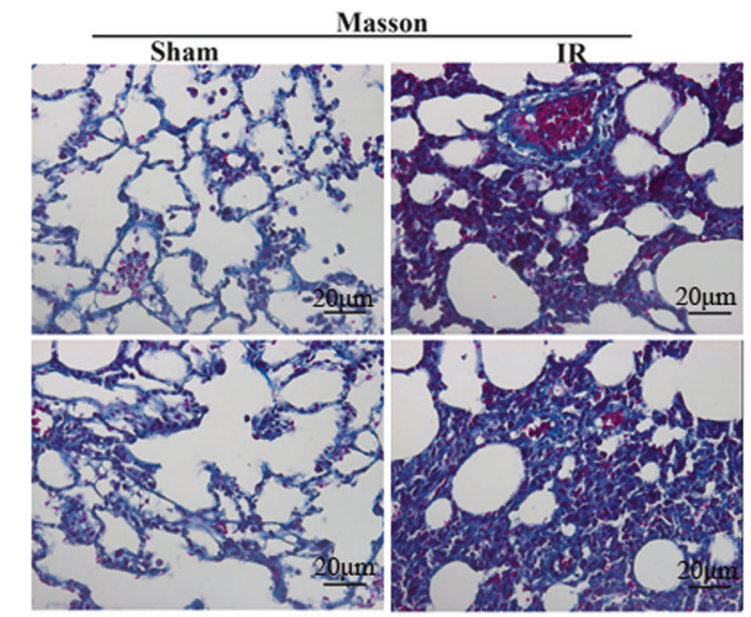

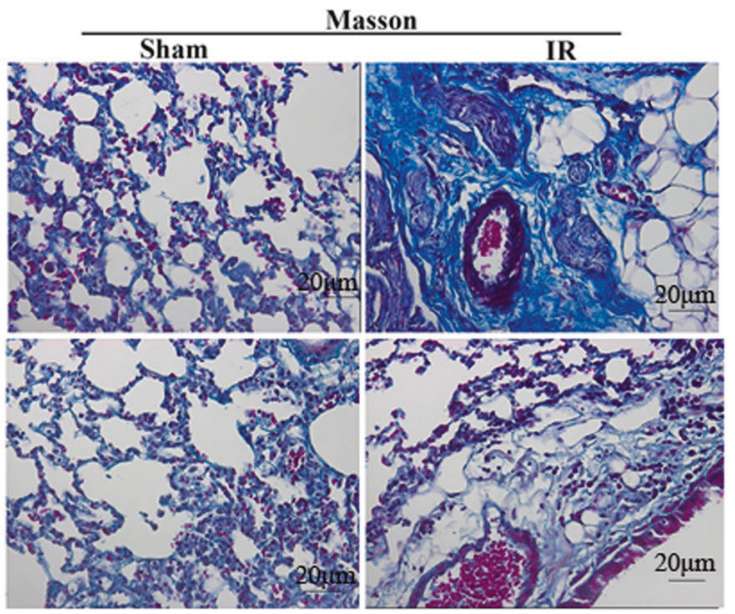

following $\mathrm{I} / \mathrm{R}(60 \mathrm{~min} / 60 \mathrm{~min})$. Scar bar $=20 \mu \mathrm{m}$. e Representative $\mathrm{H} \& \mathrm{E}-$ and Masson trichrome-stained lung sections. Morphology was examined using light microscopy, WT mice received adenoviruses carrying si-iASPP or si-Ctrl by injection. f Representative H\&E- and Masson trichrome-stained lung sections. Morphology was examined using light microscopy. WT mice received adenoviruses carrying vector control or iASPP by injection. ${ }^{*} p<0.01,{ }^{* * *} p<0.001$. 
A

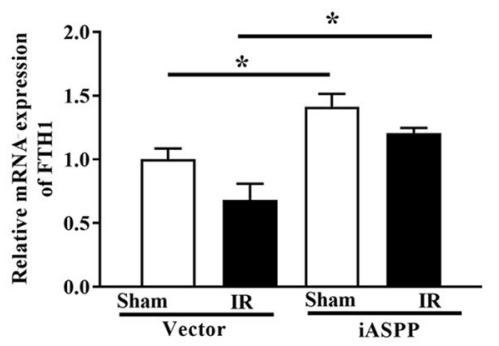

D

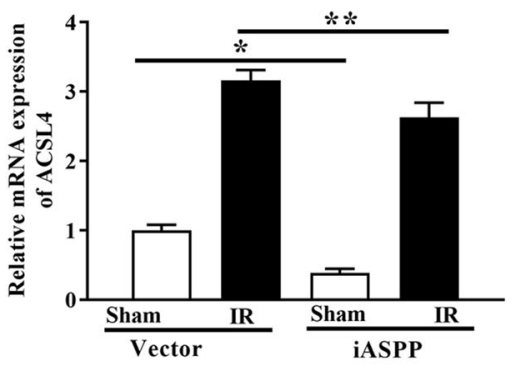

F

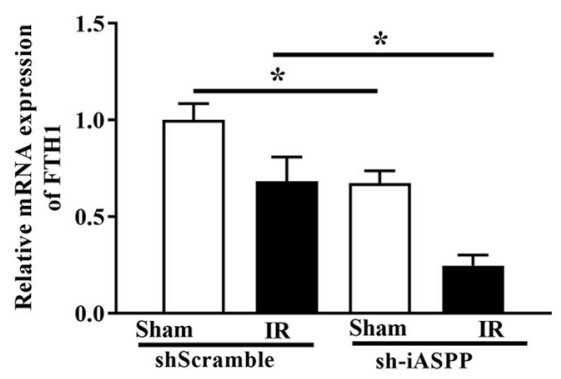

I

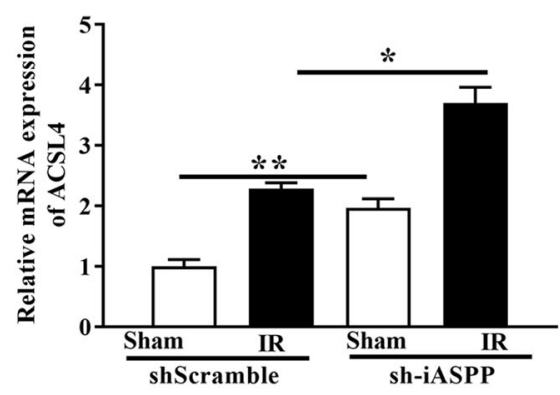

B

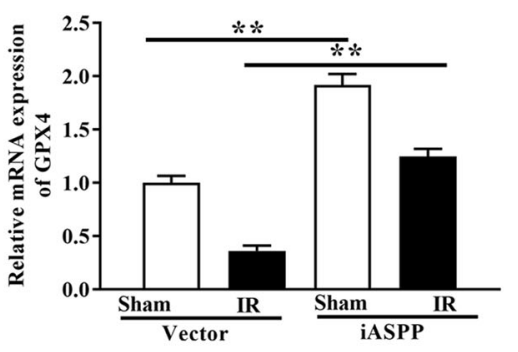

C

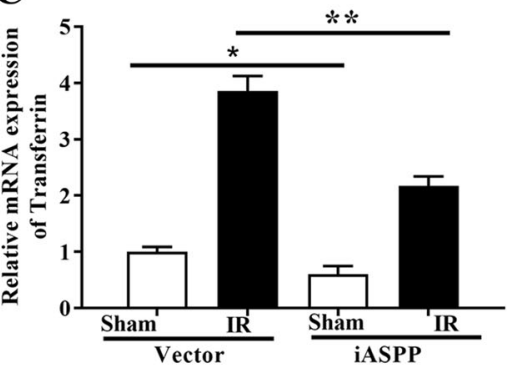

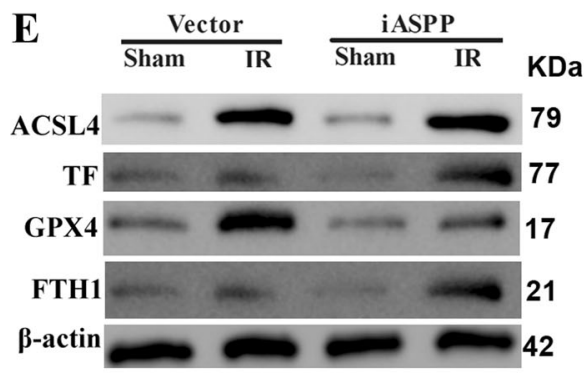

G

H
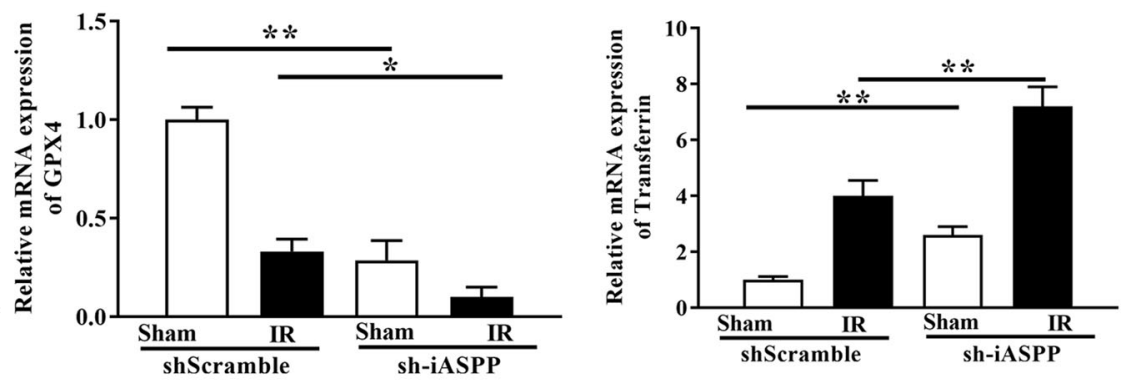

$\mathbf{J}$

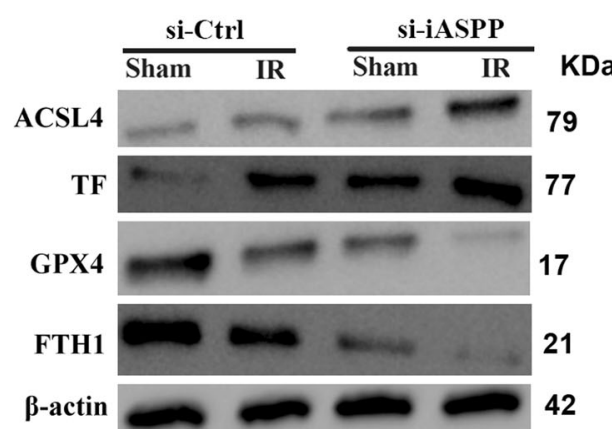

Fig. 4 Effect of iASPP knockdown or overexpression on ferroptosis in vivo. a-d The mRNA expression levels for FTH1, GPX4, TF, and ACSL4 following I/R (60 $\mathrm{min} / 60 \mathrm{~min})$ were detected by RT-PCR. WT mice received adenoviruses carrying vector control or iASPP by injection. e Relative protein expression levels of FTH1, GPX4, TF, and ACSL4 following I/R (60 $\mathrm{min} / 60 \mathrm{~min})$ as assessed by western blot analysis. WT mice received adenoviruses carrying vector control or
iASPP by injection. f-i mRNA expressions of FTH1, GPX4, TF, NQO-1, and HO-1 following I/R $(60 \mathrm{~min} / 60 \mathrm{~min})$ were detected by RT-PCR. WT mice received adenoviruses carrying si-iASPP or si-Ctrl by injection. $\mathbf{j}$ Relative protein expressions of FTH1, GPX4, TF, and ACSL4 following I/R $(60 \mathrm{~min} / 60 \mathrm{~min})$ as assessed by western blot analysis. WT mice received adenoviruses carrying si-iASPP or si-Ctrl by injection. $* p<0.05, * * p<0.01$. 
Nrf2 deficiency alters the effect of I/R on ALI. iASPP overexpression had no effect on Nrf2 mRNA expression but resulted in increased Nrf2 protein levels (Fig. 5a, b). iASPP inhibition decreased Nrf2 protein but not mRNA expression (Fig. 5c, d). Notably, there was a difference between mRNA and protein iASPP expression. These data suggest that iASPP induces Nrf2 accumulation, and that this effect is unlikely to occur at the transcriptional level. These findings might be explained by the interaction of iASPP with Keap1, which is affected by protein levels or by the tightly controlled expression of iASPP at the posttranslational level. Therefore, we postulated that iASPP might elevate Nrf2 by regulating its protein stability. The expression of iASPP and Nrf2 proteins significantly increased in the lungs of WT mice $60 \mathrm{~min}$ after I/R. In contrast, Nrf2 was absent, and iASPP levels were lower in $\mathrm{Nrf2^{-/- }}$ I/R mice than WT mice (Fig. 5e).

To further assess the role of iASPP in intestinal I/Rinduced ALI, we performed H\&E and double immunohistochemical staining of lung tissues after $\mathrm{I} / \mathrm{R}(60 \mathrm{~min} / 60 \mathrm{~min})$ in WT mice and $\mathrm{Nrf}^{-/-}$mice. Histological examination and pathological scoring of lung tissues from WT and $\mathrm{Nrf}^{-/-}$I/R mice receiving adenovirus encoding iASPP or the vector control showed that edema, atelectasis, necrosis, alveolar and interstitial inflammation, and pulmonary fibrosis were aggravated in $\mathrm{Nrf}^{-/-}$mice and that iASPP overexpression diminished the pathological findings (Fig. 5f). Double immunohistochemical staining of lung tissues showed higher Nrf2 (green) and iASPP (red) levels in $\mathrm{I} / \mathrm{R}$ mice than sham mice (Fig. $5 \mathrm{~g}$ ). Nuclei were stained with DAPI (blue). These data indicated that iASPP is critical to maintaining Nrf2 stability and iASPP-mediated intestinal I/R-induced ALI is dependent on Nrf2 signaling in vivo.

\section{Ferroptosis is enhanced by intestinal I/R-induced ALI in $\mathrm{Nrf2}^{-I-}$ mice compared with WT mice}

The relative values of oxidative stress markers (GSH, $\mathrm{MDA}$, and $\mathrm{Fe}^{2+}$ ) were assessed. The percentage GSH significantly decreased, and the percentage MDA and $\mathrm{Fe}^{+}$ levels significantly increased (Fig. 6a-c). Examination of ferroptosis-related proteins (FTH1, GPX4, TF, NQO-1, and HO-1) (Fig. 6d-i) indicated that ferroptosis and oxidative stress were significantly greater in $\mathrm{Nrf}^{-/-}$mice than in WT mice. In conclusion, Ferroptosis is more severe in $N r f 2^{-/-}$ mice compared with WT mice.

\section{Nrf2 deficiency upregulates HIF-1a and TF, and promotes $\mathrm{I} / \mathrm{R}$-induced $\mathrm{ALI}$ in vivo}

H\&E- and Masson-stained lung tissues (Fig. 7a) and pathological scores (Fig. 7b) showed more pronounced pathological findings in WT I/R, and $N r f 2^{-/-} \mathrm{I} / \mathrm{R}$ and $N r f 2^{-1-}$ sham mice than in WT sham controls.

IL-1 $\beta$, IL-6, and TNF- $\alpha$ levels were significantly higher in the BALF of $\mathrm{Nrf}^{-/-}$sham mice than in WT sham mice, and these effects were significantly enhanced in $\mathrm{Nrf}^{-2^{--}}$IR mice compared with those in WT I/R mice (Fig. S3A-C). The mRNA/protein expression of HIF- $1 \alpha$ and TF was significantly higher in WT I/R mice than in WT sham controls and in $N r f 2^{-/-}$I/R mice than in $N r f 2^{-/-}$sham controls (Fig. 7f, g). Together, these data demonstrating that Nrf2 expression is vital for I/R-induced ALI in vivo.

\section{HR-induced ALI promotes ferroptosis in MLE-2 cells}

Subsequently, we conducted in vitro experiments on MLE-2 cells to assess the relationship between hypoxia/ regeneration (HR)-induced ALI and ferroptosis. The protein and mRNA expression of FTH1 and GPX4 significantly decreased, and that of ACSL4 and TF significantly decreased, in MLE-2 cells after $2 \mathrm{~h}$ of hypoxia and $0,3,6$, or $12 \mathrm{~h}$ of regeneration (Fig. 8a-d). An increase in regeneration time enhanced the effects on protein and mRNA expression. The protein and mRNA expression of ACSL4 and TF, increased, and that of FTH1 and GPX4 decreased, in the HR + erastin ( $10 \mu \mathrm{M}$ for $24 \mathrm{~h})$ group compared with the HR alone and control groups (Fig. 8e-i). The addition of liproxstatin-1 (1 $\mu \mathrm{M}$ for $24 \mathrm{~h})$ reversed the effect of erastin to levels similar to those of the HR alone group.

\section{iASPP knockdown enhances ferroptosis, and iASPP overexpression inhibits ferroptosis in MLE-2 cells}

The protein and mRNA expression of iASPP was significantly higher in MLE- 2 cells transfected with adenovirus encoding iASPP than with the vector control (Fig. S4A). Figure S4B shows the significant increase in iASPP in the cytoplasmic fraction compared with the nuclear fraction in MLE- 2 cells after iASPP overexpression by adenovirus, as compared with the vector control. The mRNA expression of iASPP was significantly lower in MLE-2 cells transfected with adenovirus encoding si-iASPP than si-Ctrl (Fig. S4C). The iASPP levels were lower in the cytoplasmic fraction than the nuclear fraction of MLE-2 cells after iASPP knockdown, as compared with the results in the si-Ctrl group (Fig. S4D). Immunocytochemical iASPP (red) staining was greater in MLE- 2 cells after $2 \mathrm{~h}$ of hypoxia and $12 \mathrm{~h}$ of regeneration than in untreated MLE-2 cells (Fig. S4E).

The protein levels of iASPP but not Nrf2 were decreased by iASPP knockdown in MLE-2 cells, as compared with the results in the si-Ctrl group (Fig. S4F). The addition of MG132, a proteasome inhibitor, had no 

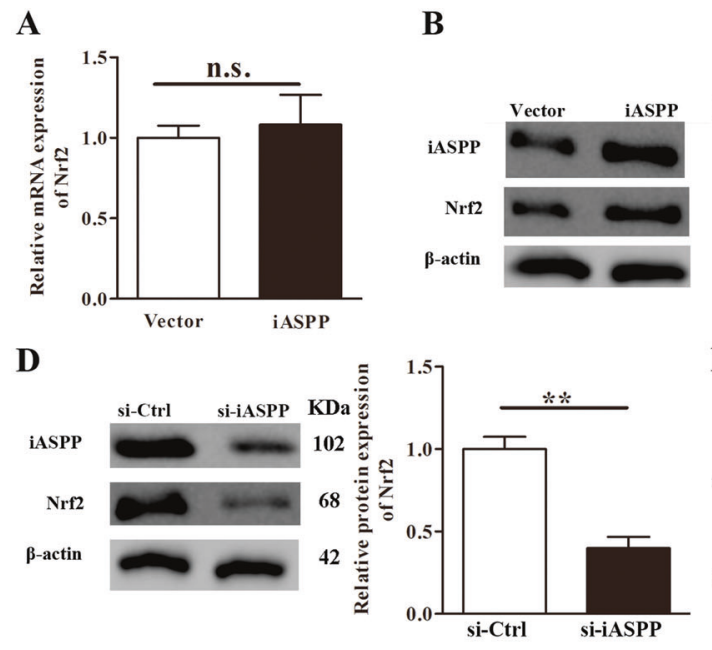

\section{B}

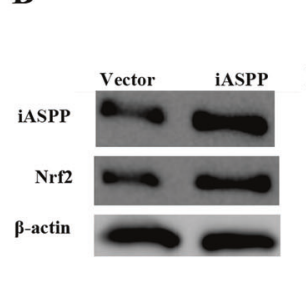

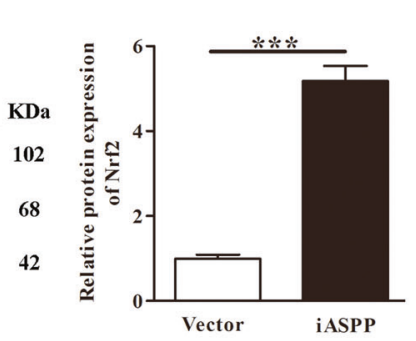

$\mathbf{E}$

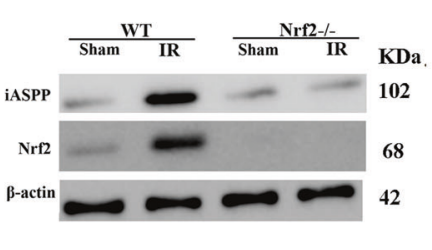

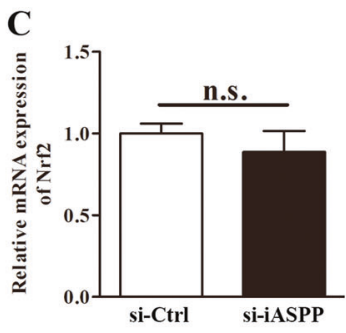

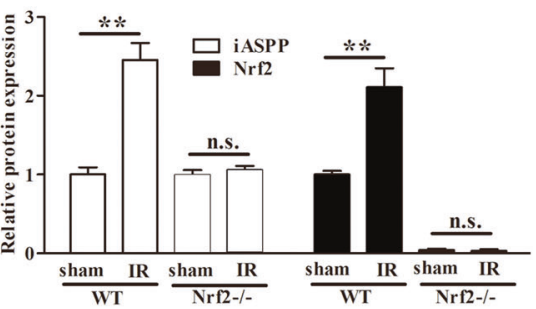

F
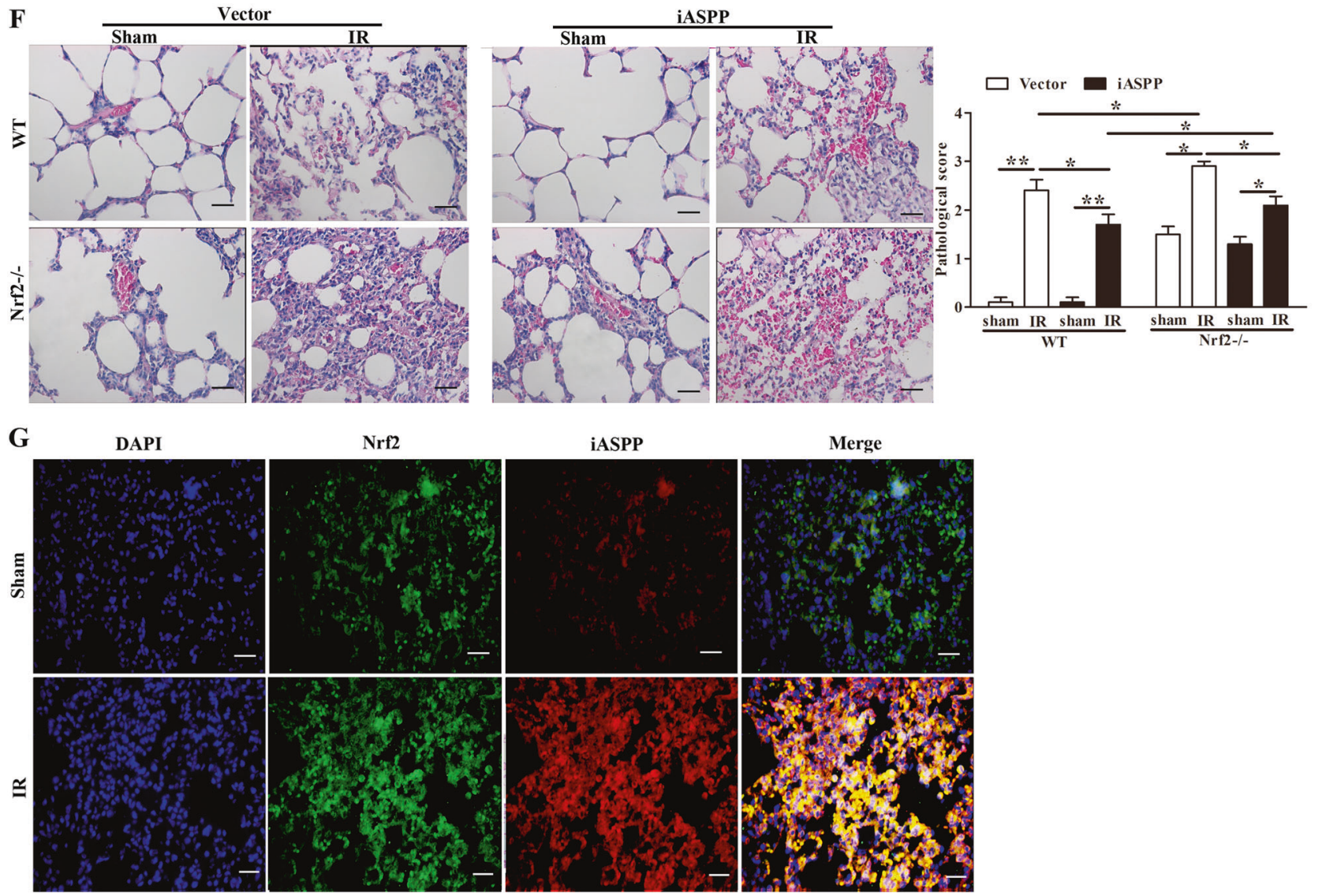

Fig. 5 iASPP-mediated protection against I/R-induced ALI is dependent on Nrf2 antioxidative signaling in vivo. The mRNA (a) and protein (b) expression of Nrf2 in lung tissues were detected by RT-PCR and western blot analysis, respectively. WT mice received adenoviruses carrying vector control or iASPP by injection. The mRNA (c) and protein (d) expression of Nrf2 in lung tissues were detected by RT-PCR and western blot analysis, respectively. WT mice received adenoviruses carrying si-iASPP or si-Ctrl by injection. e The protein expression levels of iASPP and Nrf2 in lung tissues were determined by western blot analysis in WT mice and $\mathrm{Nrf}^{-/-}$mice following I/R (60 min/60 min). f Representative H\&E staining of lung tissues following I/R $(60 \mathrm{~min} / 60 \mathrm{~min})$ in WT mice and $\mathrm{Nrf2^{-/- }}$ mice. WT mice and $\mathrm{Nrf} 2^{-/-}$mice received adenoviruses carrying vector control or iASPP by injection. Scar bar $=20 \mu \mathrm{m}$. g Double immunohistochemical staining of lung tissues for Nrf2 (green) and iASPP (red). Scar bar $=20 \mu \mathrm{m}$. Nuclei were stained with DAPI (blue) following $\mathrm{I} / \mathrm{R}(60 \mathrm{~min} / 60 \mathrm{~min}) .{ }^{*} p<0.05, * * p<0.01$. 
A
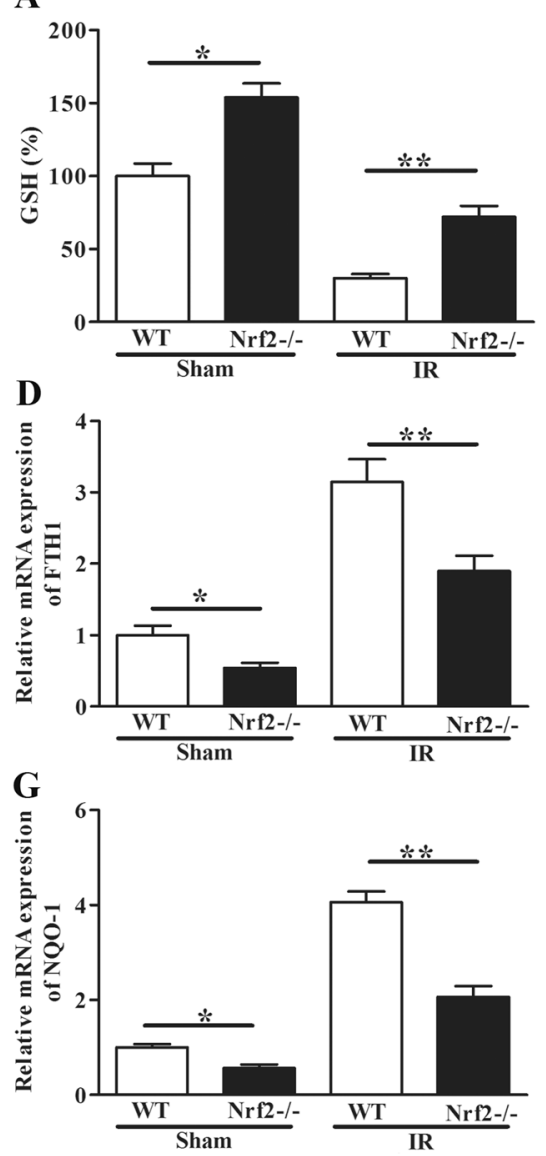

B

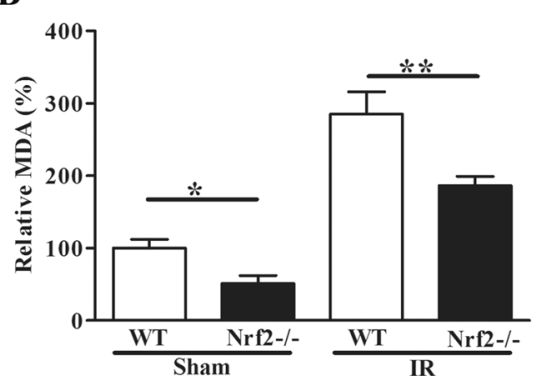

E

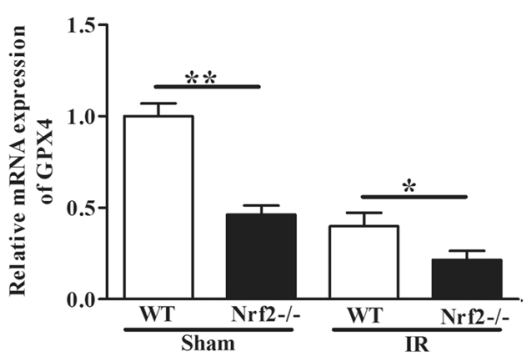

H

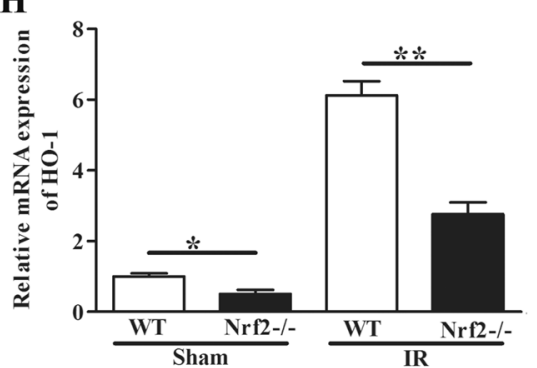

C
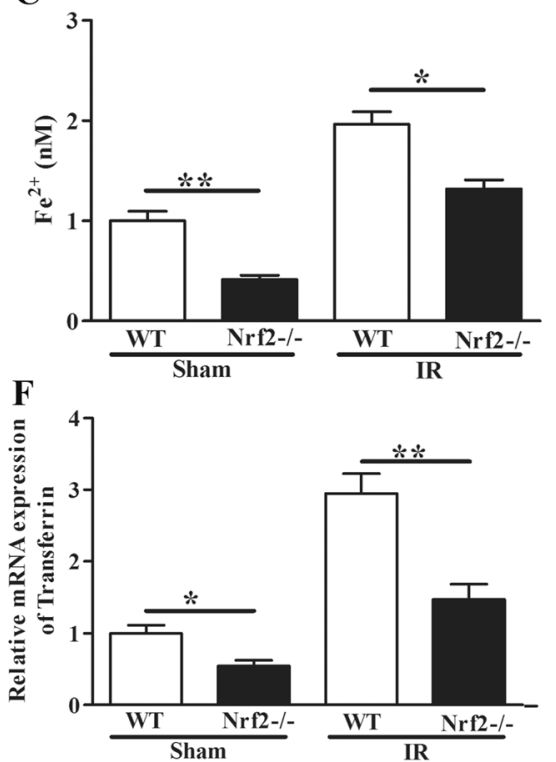

I

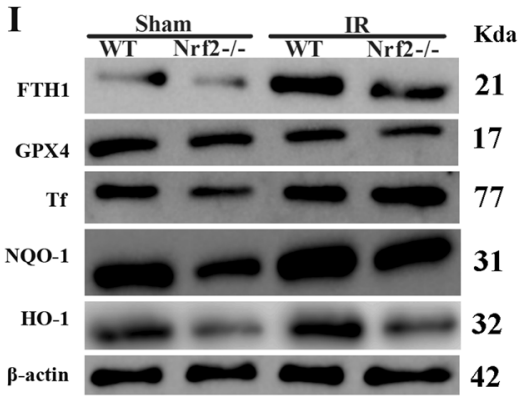

Fig. 6 Modulation of ferroptosis is impaired in $\mathrm{Nrf2}^{-/-}$mice after intestinal I/R. The relative values of GSH (a) and MDA (b) and the $\mathrm{Fe}^{2+}$ concentration (c) were measured following I/R (60 min/60 min) in WT and $\mathrm{Nrf}^{-/-}$mice. d-h The mRNA expression levels for FTH1, GPX4, TF, NQO-1, and HO-1 following I/R (60 $\mathrm{min} / 60 \mathrm{~min})$ were detected by RT-PCR in WT mice and $\mathrm{Nrf}^{-/-}$mice. $\mathbf{i}$ The protein expression levels of FTH1, GPX4, TF, NQO-1, and HO-1 following $\mathrm{I} / \mathrm{R}(60 \mathrm{~min} / 60 \mathrm{~min})$ were detected by western blot analysis in WT mice and $N r f 2^{-1-}$ mice. ${ }^{*} p<0.05, * * p<0.01$. effect on iASPP levels but enhanced the levels of Nrf2 in both groups (Fig. S4F). The protein levels of FTH1 and GPX4 decreased (and those of ACSL4 increased) after HR in MLE-2 cells, and these effects were diminished when iASPP was overexpressed (Fig. S4G) or enhanced after iASPP knockdown (Fig. S4H). Overall, our results indicated that the inhibition of iASPP induces ferroptosis in MLE-2 cells.

\section{iASPP alleviates ALI and ferroptosis through the Nrf2/HIF-1a/TF signaling pathway in vitro}

We examined the expression of Nrf2/HIF-1 $\alpha /$ TF in MLE2 cells after HR under the conditions of interference or overexpression. The results in Fig. 9a indicated that the protein levels of iASPP, Nrf2, and HIF-1 $\alpha$, but not TF, increased in MLE- 2 cells after HR, and these effects were decreased after iASPP knockdown or enhanced after iASPP overexpression. In addition, Nrf2 levels increased in the nuclear but not the cytoplasmic fractions of MLE-2 cells after iASPP overexpression (Fig. 9b). We then detected the distribution of Nrf2 in MLE-2 cells by immunofluorescence, and histochemical analysis showed greater Nrf2 staining (green) in the nuclei of MLE-2 cells overexpressing iASPP for $24 \mathrm{~h}$ before HR induction than in control cells (Fig. 9c). Double immunofluorescence staining of Nrf2 (green) and iASPP (red) in untreated MLE-2 cells was enhanced after $2 \mathrm{~h}$ hypoxia and $12 \mathrm{~h}$ regeneration (Fig. 9d). The protein level of HIF$1 \alpha$ was higher, and the Nrf2 and TF levels were lower, in MLE-2 cells treated with si-Nrf2 than with si-Ctrl (Fig. 9e). The mRNA expression of FTH1, HO-1, TF, and NQO-1 increased, and GPX4 decreased, in MLE-2 cells overexpressing iASPP, but these effects were reversed in cells treated with si-Nrf2 but not si-Ctrl (Fig. 9f-j). These data indicate that iASPP alleviates ALI and ferroptosis through the Nrf2/HIF-1 $\alpha /$ TF signaling pathway in vitro. 


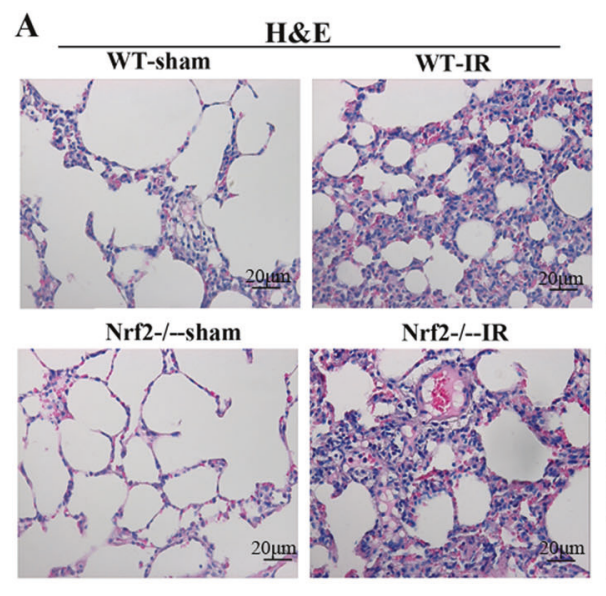

C

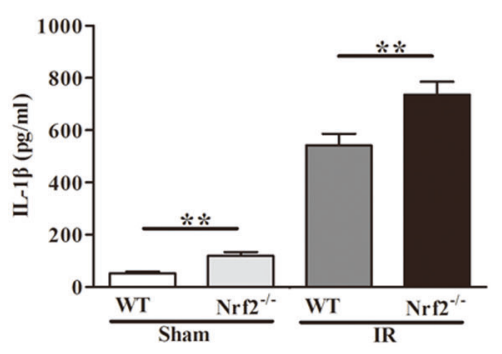

F

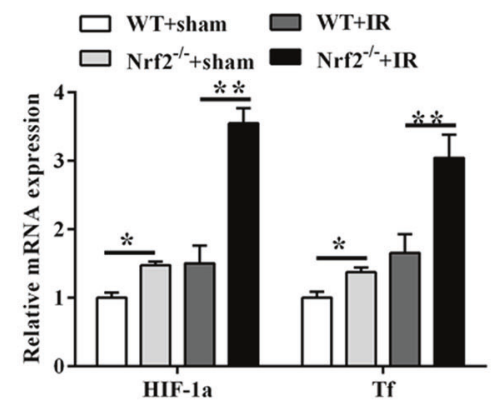

G

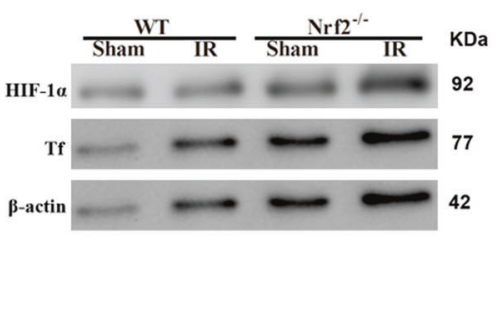

D
B
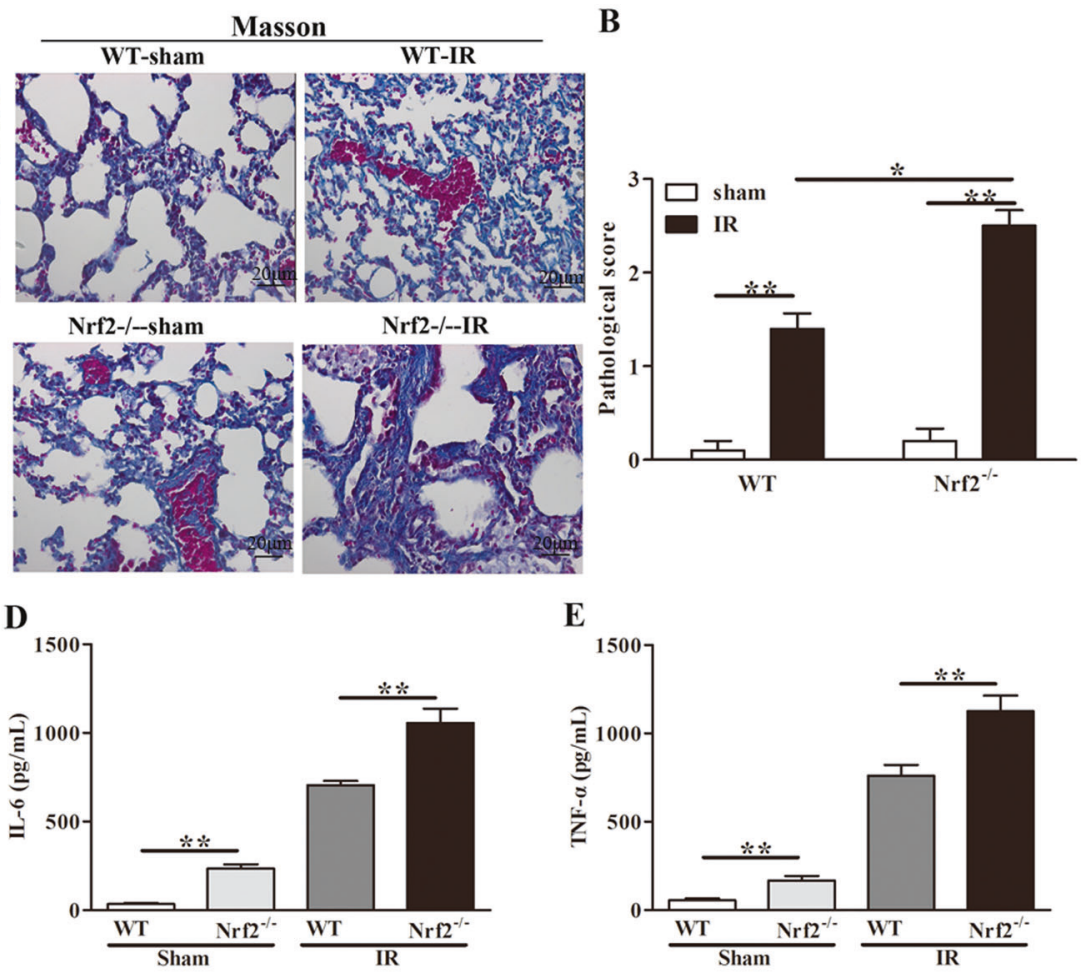

$\mathbf{E}$
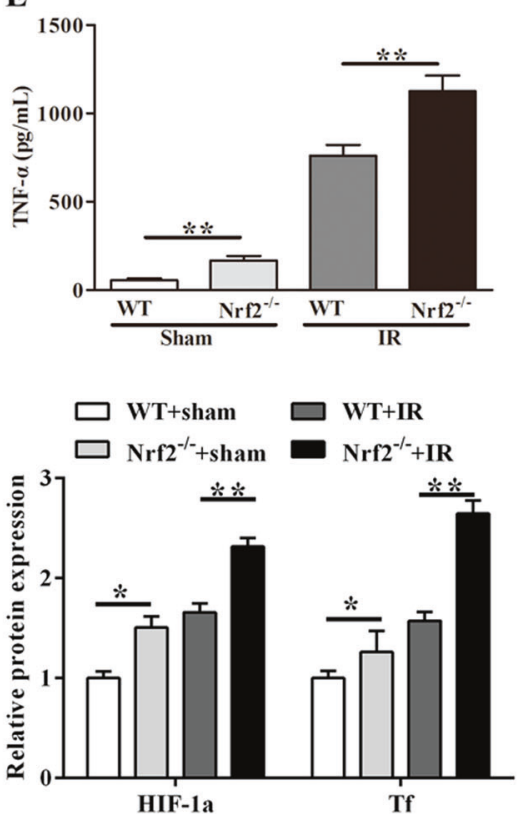

Fig. 7 Nrf2 regulates I/R-induced ALI through the HIF-1 $\alpha /$ TF signaling pathway in vivo. a, b Representative H\&E- and Masson trichrome-stained lung tissues were detected following I/R (60 min/ $60 \mathrm{~min}$ ) in WT mice and $\mathrm{Nrf}^{-/-}$mice. Morphology was examined using light microscopy. Pathological scores were assigned by an experienced pathologist. c-e Concentrations of IL-1 $\beta$, IL-6, and TNF- $\alpha$ were measured in BALF by ELISA following I/R $(60 \mathrm{~min} / 60$ min) in WT mice and $N r f 2^{-/-}$mice. $\mathbf{f}$ The mRNA expression levels for HIF- $1 \alpha$ and TF were determined by RT-PCR following I/R $(60 \mathrm{~min} / 60 \mathrm{~min})$ in WT mice and $N r f 2^{-/-}$mice. $\mathrm{g}$ The protein expressions of HIF- $1 \alpha$ and TF were detected by western blot analysis following I/R $(60 \mathrm{~min} / 60 \mathrm{~min})$ in $\mathrm{WT}$ and $\mathrm{Nrf2^{-1- }}$ mice. $* p<0.05, * * p<0.01$.

\section{Discussion}

This study evaluated the roles of iASPP and Nrf2 in ferroptosis and their potential therapeutic effects on intestinal I/R-induced ALI in vivo and in vitro. The major findings of the study are as follows: (1) ferroptosis contributes to intestinal I/R-induced ALI in vivo; (2) iASPP inhibits ferroptosis and alleviates intestinal I/R-induced ALI; (3) iASPP-mediated protection against I/R-induced ALI depends on Nrf2 signaling; (4) HR-induced ALI enhances ferroptosis in vitro; (5) the modulation of ferroptosis after intestinal I/R is enhanced in $N r f 2^{-/-}$mice; and (6) iASPP mediates its protective effects against ALI through the Nrf2/ HIF-1/TF signaling pathway.

Ferroptosis is an iron-dependent, non-apoptotic form of cell death characterized by intracellular accumulation of ROS, and it is morphologically, biochemically, and genetically distinct from autophagy, apoptosis, and necrosis [24]. Although the physiological function of ferroptosis has not been defined, its role in human diseases has been established. Ferroptosis has emerged as a new form of regulated necrosis that has been implicated in various 


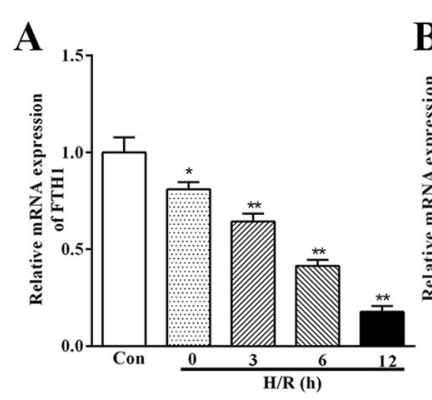

$\mathbf{E}$

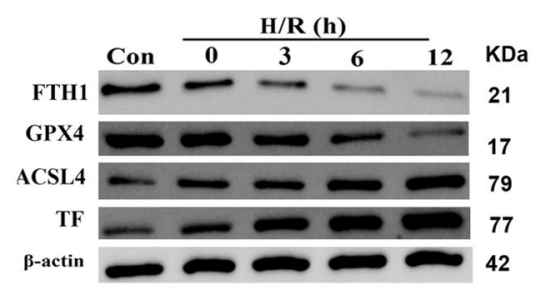

H

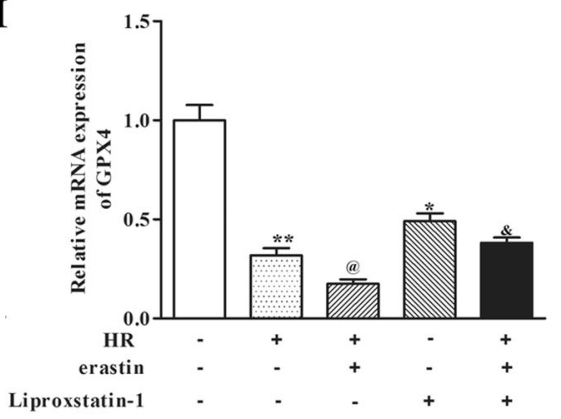

Fig. 8 HR-induced ALI enhances ferroptosis in vitro. Mouse lung epithelial (MLE-2) cells were subjected to $2 \mathrm{~h}$ of hypoxia followed by $0,3,6$, or $12 \mathrm{~h}$ of regeneration. a-d The mRNA expression levels for FTH1, GPX4, NQO-1, and HO-1 were determined by RT-PCR following HR in MLE-2 cells. e The protein expression levels of FTH1, GPX4, NQO-1, and HO-1 were determined by western blot analysis

human diseases. In this study, we showed that ferroptosis enhances intestinal I/R-induced ALI. This finding is in line with results from previous studies suggesting that ferroptosis is involved in the pathogenesis of human diseases such as Huntington's disease, periventricular leukomalacia, and kidney dysfunction [25], as well as I/R injury [13, 25] and infectious diseases [26]. Moreover, ferroptosis has been reported to be involved in ischemia-induced organ injury, and inhibition of ferroptosis has been shown to be effective in treating I/R-induced organ damage in multiple experimental models $[14,22]$.

iASPP is a known inhibitor of p53 transcriptional activity that can be used to understand whether iASPP is involved in ALI. We have demonstrated that iASPP treatment inhibits ferroptosis and alleviates intestinal I/R-induced ALI, thus confirming findings from previous studies reporting the
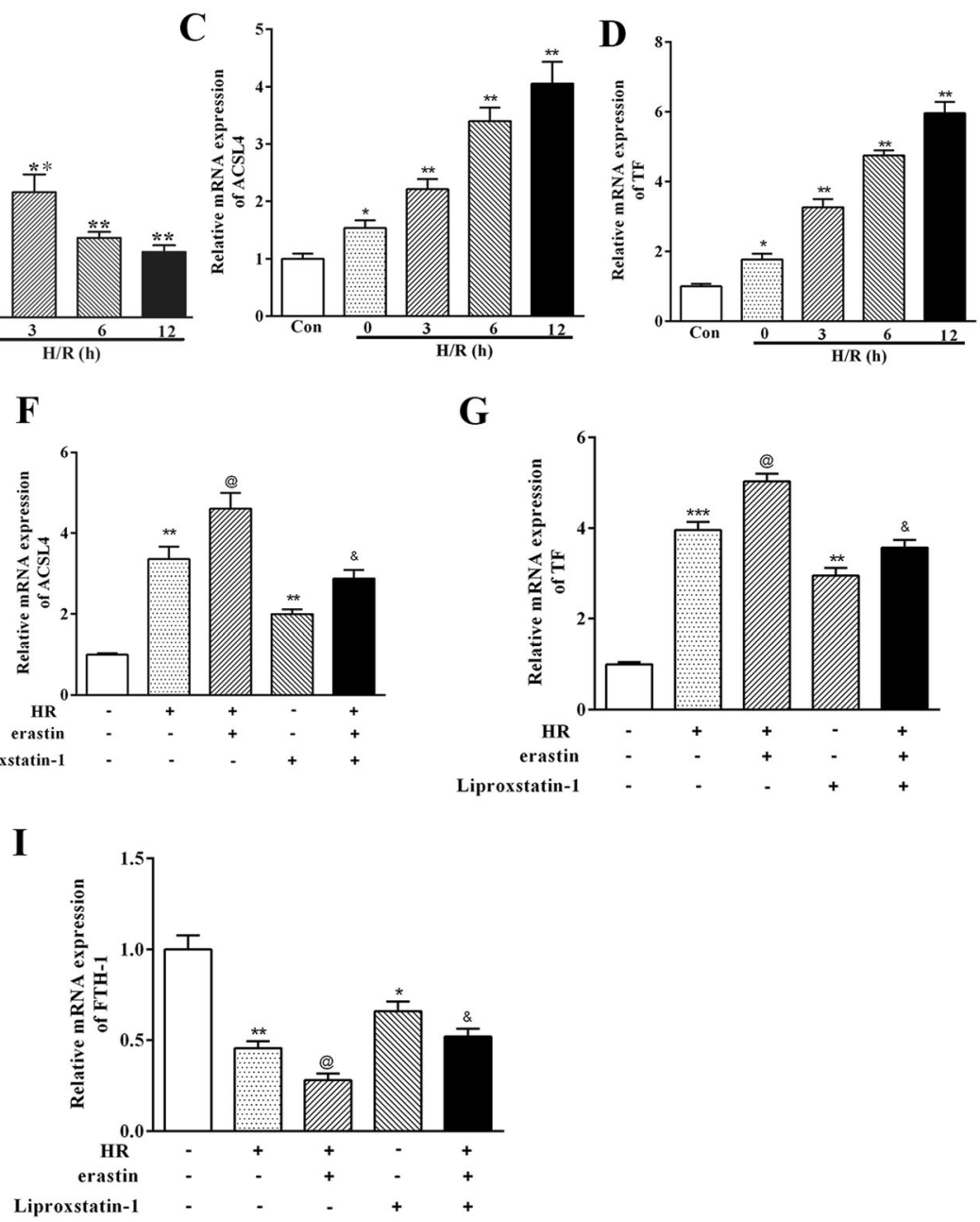

following HR in MLE-2 cells. $\mathbf{f}-\mathbf{i}$ The mRNA expression levels for FTH1, GPX4, NQO-1, and HO-1 were determined by RT-PCR following HR $(2 \mathrm{~h} / 12 \mathrm{~h})$ in MLE- 2 cells, and then MLE- 2 cells were treated with erastin $(10 \mu \mathrm{M})$ for $24 \mathrm{~h}$, followed by treatment with liproxstatin-1 $(1 \mu \mathrm{M})$ for $24 \mathrm{~h} * p<0.05, * * p<0.01, * * * p<0.001$, ${ }^{\circledR} p<0.05,{ }^{\&} p<0.05$.

protective role of iASPP in other models of $\mathrm{I} / \mathrm{R}$-mediated injury. For example, hypothermia can protect against I/R brain injury and apoptosis in mice via the upregulation of iASPP [27], and cerebral ischemia injury is aggravated by the inhibition of iASPP [28].

A previous study has demonstrated iASPP/Nrf2 mediated antiapoptotic effects, and indicated that the identified iASPP/Nrf2 axis plays an important role in both cell growth and drug responses [29]. The current study demonstrates the importance of Nrf2 in I/R-induced ALI. We found that ferroptosis is enhanced by intestinal $\mathrm{I} / \mathrm{R}$-induced ALI in $\mathrm{Nrf}^{-/-}$mice, that Nrf2 deficiency in $\mathrm{Nrf2}^{-/-}$mice promoted I/R-induced ALI, and that iASPP-mediated protection against I/R-induced ALI is Nrf2 dependent. These findings confirm previous reports of the role of Nrf2 in protection against I/R injury [18]. Other studies have shown 
A

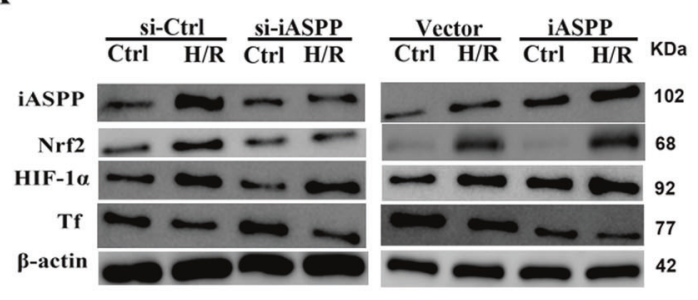

C
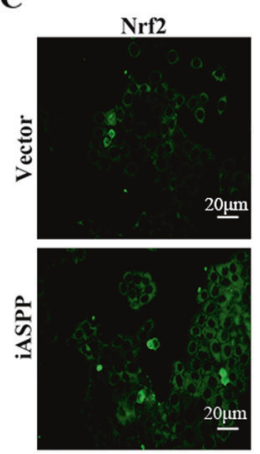

E
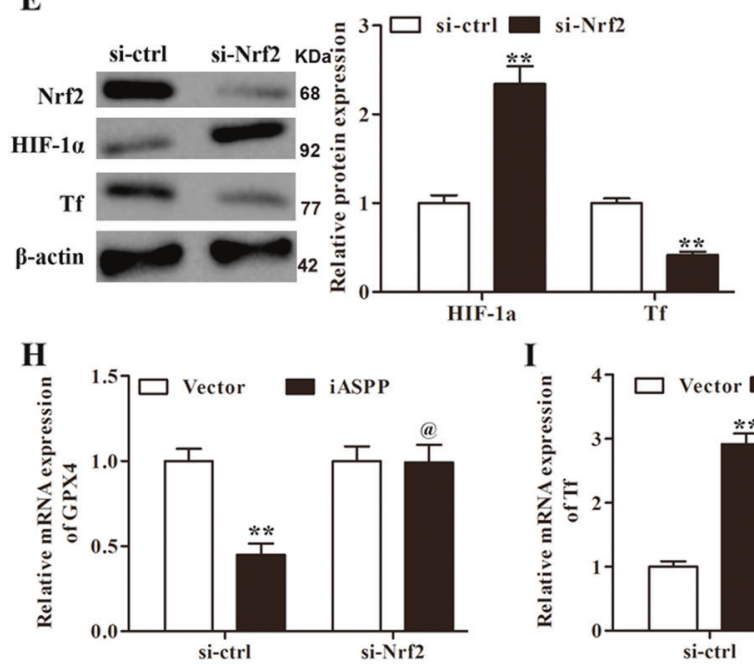

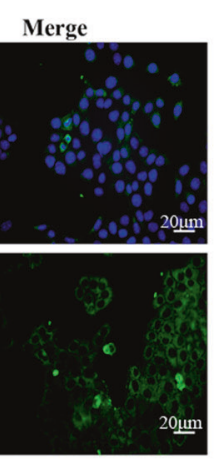

(1)
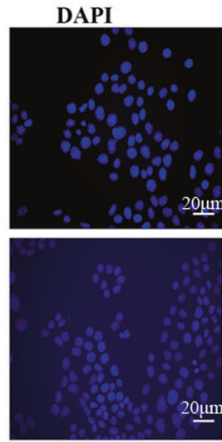

$$
\text { I }
$$

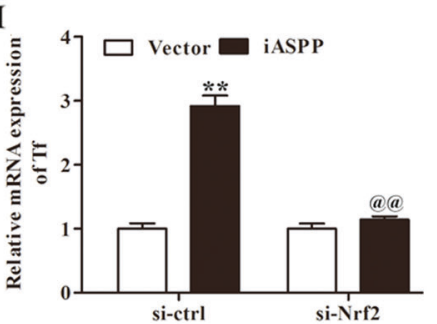

B

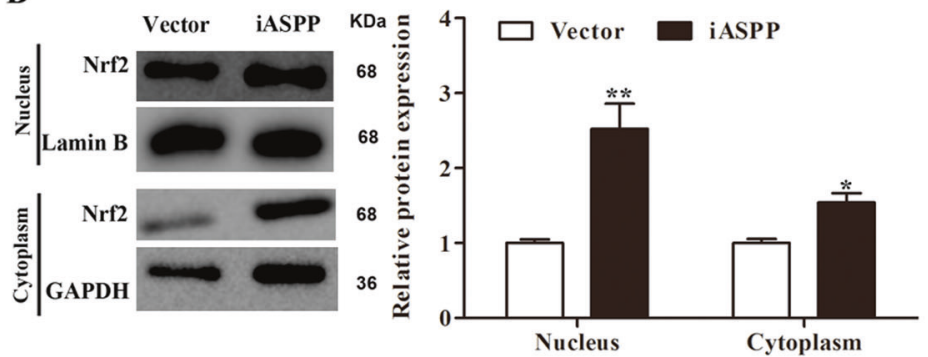

D
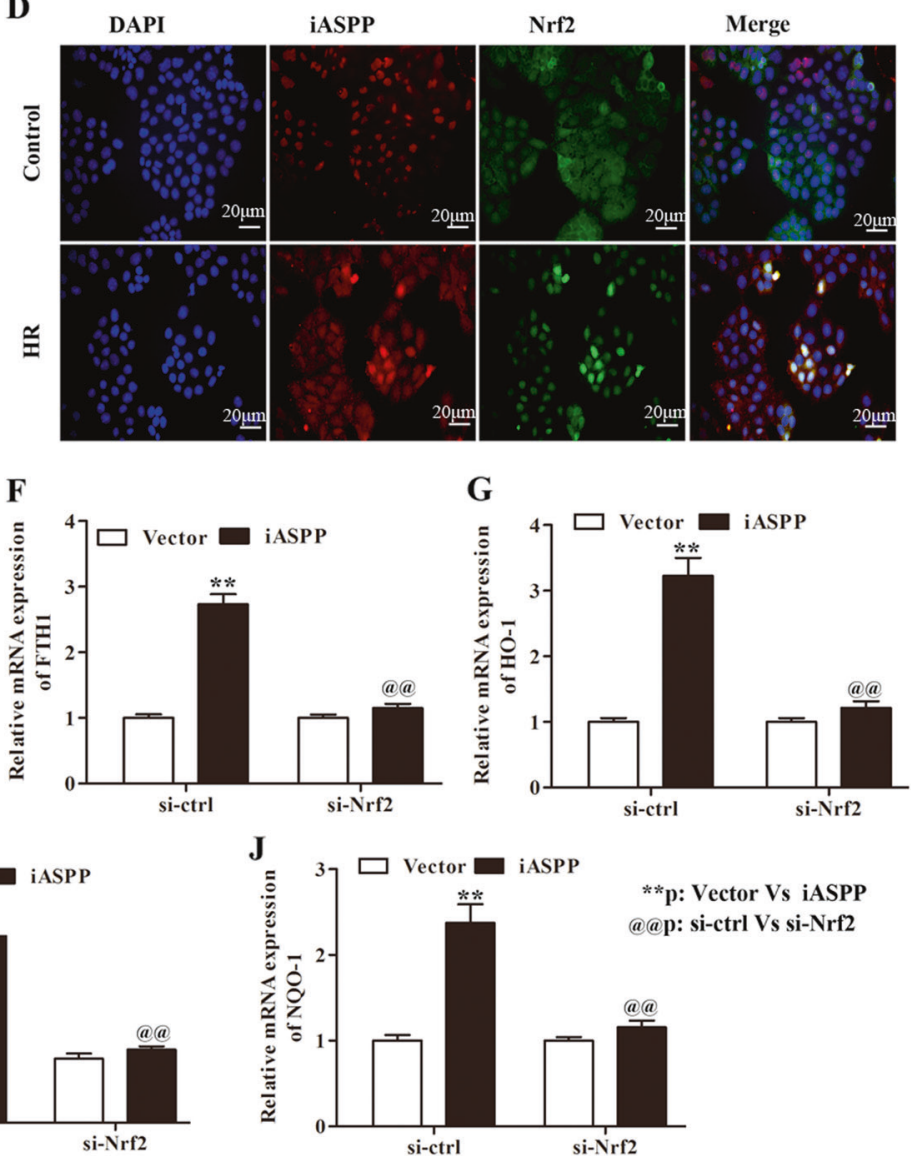

Fig. 9 iASPP regulates ALI and ferroptosis through the Nrf2/HIF-

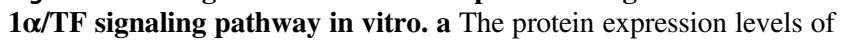
iASPP, Nrf2, HIF-1 $\alpha$, and TF were determined by western blot analysis following HR in MLE-2 cells after iASPP knockdown or iASPP overexpression. b The distribution of Nrf2 was determined by examining nuclear and cytoplasmic fractions from MLE-2 cells after iASPP overexpression. c MLE- 2 cells were transfected with vector or iASPP for $24 \mathrm{~h}$ before HR. The distribution of Nrf2 was evaluated by

a that Nrf2 protects against iron toxicity of liver parenchymal cells in vitro and in vivo [30], that the Keap1Nrf2 complex prevents oxidative injury in I/R-stressed rats [31], that IL-18 protects against lipopolysaccharide-induced ALI via Nrf2 [32], and that sinomenine protects against Escherichia coli-induced ALI in mice through Nrf2 [33].

We also investigated the roles of other ferroptosis-related molecules. We found that the mRNA expression of GPX4, immunofluorescence staining. d The co-localization of iASPP (red) and Nrf2 (green) was visualized by immunofluorescence staining. Nuclei were stained with DAPI (blue) following HR $(2 \mathrm{~h} / 12 \mathrm{~h})$. e The protein expression levels of HIF-1 $\alpha$ and TF were determined by western blot analysis in MLE-2 cells treated with si-ctrl or si-Nrf2. $\mathbf{f}-\mathbf{j}$ The mRNA expression levels for FTH1, HO-1, GPX4, TF, and NQO1 were detected by RT-PCR in MLE-2 cells treated with si-ctrl or siNrf2. $* p<0.05, * * p<0.01, \stackrel{@}{p} p<0.05,{ }^{@} @ p<0.01$.

a phospholipid hydroperoxidase that protects cells against membrane lipid peroxidation and controls ferroptosis [34], was increased by liproxstatin-1 treatment and correlated with diminished pathological findings in $\mathrm{I} / \mathrm{R}$ mice treated with erastin and liproxstatin-1 (Fig. 2). This finding is in agreement with a previous study showing that the GSH/ Gpx4 axis prevents lipid oxidation-induced acute renal failure and associated death [13]. Another study has shown 
that $\mathrm{TF}$ modulates animal models of $\mathrm{I} / \mathrm{R}$ injury [35], thus supporting our finding that iASPP mediates its protective effects against ALI in part through the Nrf2/HIF-1/TF signaling pathway.

The current study also confirmed the roles of proinflammatory cytokines in ALI. iASPP overexpression decreased the levels of TNF- $\alpha$, IL- $1 \beta$, and IL- 6 in the BALF and diminished I/R-induced ALI-findings similar to those in a previous study in which Chuanxiong Kangxian granules were found to have a beneficial effect on bleomycininduced pulmonary fibrosis in rats by decreasing the levels of TNF- $\alpha$, IL- $1 \beta$, and IL- 6 in the BALF and attenuating bleomycin-induced oxidative stress and inflammation [36].

iASPP was mainly expressed in the cytoplasm, and iASPP overexpression promoted Nrf2 accumulation and nuclear translocation, which has been shown to be protective against numerous airway disorders/diseases including ALI [18]. On the basis of the findings of the current study and previous reports, we propose that iASPP-induced protection is depending on Nrf2 expression, thereby increasing the expression of HIF-1 $\alpha$; decreasing TF and the ferroptosis-related proteins FTH1, NQO-1, and HO-1; and increasing GPX4 expression, as demonstrated in MLE2 cells.

We found that erastin, a small molecule that promotes ferroptosis, significantly depleted intracellular GSH, thus contributing to lipid peroxidation, ferroptosis, and the loss of protective GPX4 expression, thereby confirming results from a previous study in which genetic or drug approaches have been found to promote ferroptosis through a lipid ROS-dependent mechanism [37]. Interestingly, liproxstatin1 reversed this effect, thus suggesting that it might have potential for therapeutic application.

Our in vitro study using MLE-2 cells showed that HRinduced ALI enhanced ferroptosis, iASPP overexpression inhibited ferroptosis, and iASPP alleviated ALI and ferroptosis through the Nrf2/HIF-1 $\alpha /$ TF signaling pathway.

In conclusion, our findings describe a novel mechanism for ferroptosis that involves altering iron transport in I/Rinduced ALI, and demonstrate that iASPP treatment inhibits ferroptosis via Nrf2, thus indicating its therapeutic potential for treating I/R-induced ALI.

Acknowledgements We thank J. Ludovic Croxford, PhD, of Edanz Medical Writing for providing editorial support.

Funding This study was supported by the Natural Science Foundation of Shanghai 18ZR1428800 and 81572626 .

\section{Compliance with ethical standards}

Conflict of interest The authors declare that they have no conflict of interest.
Publisher's note Springer Nature remains neutral with regard to jurisdictional claims in published maps and institutional affiliations.

\section{References}

1. Ng CSH, Wan S, Arifi AA, Yim APC. Inflammatory response to pulmonary ischemia-reperfusion injury. Surg Today. 2006;36: 205-14.

2. De-Perrot M, Liu M, Waddell T,S. Ischemia-reperfusion-induced lung injury. Am J Respir Crit Care Med. 2003;167:490-511.

3. Axelle C, Kai K, Gilliss BM, Nguyen JX, Marques MB, Marc M, et al. Platelets induce neutrophil extracellular traps in transfusionrelated acute lung injury. J Clin Investig. 2012;122:2661.

4. Ortiz MG, Mallavia B, Bins A, Headley M, Krummel MF, Looney MR. Aspirin-triggered 15-epi-lipoxin A4 regulates neutrophilplatelet aggregation and attenuates acute lung injury in mice. Blood. 2014;124:2625-34.

5. Rob Mac S, Mark G, Danny MA. Treatment of acute lung injury: current and emerging pharmacological therapies. Semin Respiratory Crit Care Med. 2013;34:487-98.

6. Johnson ER, Matthay MA. Acute lung injury: epidemiology, pathogenesis, and treatment. J Aerosol Med Pulm Drug Deliv. 2010;23:243-52.

7. Matthay MA, Ware LB, Zimmerman GA. The acute respiratory distress syndrome. J Clin Investig. 2012;122:2731-40.

8. Marco M, Andrade CF, Bing H, Rashmi S, Yu Z, Xiao HB, et al. Intestinal ischemia-reperfusion-induced acute lung injury and oncotic cell death in multiple organs. Shock. 2007;28:227-38.

9. Feng D, Yao J, Wang G, Li Z, Zu G, Li Y, et al. Inhibition of p66Shc-mediated mitochondrial apoptosis via targeting prolylisomerase Pin1 attenuates intestinal ischemia/reperfusion injury in rats. Clin Sci. 2017;131:759-73.

10. Lagan AL, Melley DD, Evans TW, Quinlan GJ. Pathogenesis of the systemic inflammatory syndrome and acute lung injury: role of iron mobilization and decompartmentalization. Am J Physiol Lung Cell Mol Physiol. 2008;294:L161.

11. Dixon S, Lemberg K, Lamprecht M, Skouta R, Zaitsev E, Gleason $\mathrm{C}$, et al. Ferroptosis: an iron-dependent form of nonapoptotic cell death. Cell. 2012;149:1060-72.

12. Andreas L, Rachid S, Nina H, Mulay SR, Christin D, Federica DZ, et al. Synchronized renal tubular cell death involves ferroptosis. Proc Natl Acad Sci USA. 2014;111:16836-41.

13. Jose Pedro FA, Manuela S, Bettina P, Tyurina YY, Tyurin VA, Hammond VJ, et al. Inactivation of the ferroptosis regulator $\mathrm{Gpx} 4$ triggers acute renal failure in mice. Free Radic Biol Med. 2014;76: 1180-91.

14. Gao M, Monian P, Quadri N, Ramasamy R, Jiang X. Glutaminolysis and transferrin regulate ferroptosis. Mol cell. 2015;59: 298-308.

15. Le J, Ning K, Tongyuan L, Shang-Jui W, Tao S, Hanina H, et al. Ferroptosis as a p53-mediated activity during tumour suppression. Nature. 2015;520:57-62.

16. Bruni A, Pepper AR, Pawlick RL, Galalopez B, Gamble AF, Kin $\mathrm{T}$, et al. Ferroptosis-inducing agents compromise in vitro human islet viability and function. Cell Death Dis. 2018;9:595.

17. Sun X, Ou Z, Chen R, Niu X, Chen, Kang R, et al. Activation of the p62-Keap1-NRF2 pathway protects against ferroptosis in hepatocellular carcinoma cells.Hepatology. 2016;63:173-84.

18. MRDL Vega, Dodson M, Gross C, Mansour HM, Lantz RC, Chapman E, et al. Role of Nrf2 and autophagy in acute lung injury. Curr Pharmacol Rep. 2016;2:91-101.

19. Kalogeris T, Baines CP, Krenz M, Korthuis RJ. Cell biology of ischemia/reperfusion injury. Int Rev Cell Mol Biol. 2012;298: 229-317. 
20. Dixon SJ, Patel DN, Welsch M, Skouta R, Lee ED, Hayano M, et al. Pharmacological inhibition of cystine-glutamate exchange induces endoplasmic reticulum stress and ferroptosis. eLife. 2014;3:e02523.

21. Nicholas Y, Moritz VR, Elma Z, Bauer AJ, Seok YW, Fridman DJ, et al. RAS-RAF-MEK-dependent oxidative cell death involving voltage-dependent anion channels. Nature. 2007;447:864-8.

22. Friedmann Angeli JP, Schneider M, Proneth B, Tyurina YY, Tyurin VA, Hammond VJ, et al. Inactivation of the ferroptosis regulator $\mathrm{Gpx} 4$ triggers acute renal failure in mice. Nat cell Biol. 2014;16:1180-91.

23. Zilka O, Shah R, Li B, Friedmann Angeli JP, Griesser M, Conrad $\mathrm{M}$, et al. On the mechanism of cytoprotection by ferrostatin- 1 and liproxstatin-1 and the role of lipid peroxidation in ferroptotic cell death. ACS Cent Sci. 2017;3:232-43.

24. Dixon SJ, Lemberg KM, Lamprecht MR, Skouta R, Zaitsev EM, Gleason CE, et al. Ferroptosis: an iron-dependent form of nonapoptotic cell death. Cell. 2012;149:1060-72.

25. Rachid S, Dixon SJ, Jianlin W, Dunn DE, Marina O, Kenichi S, et al. Ferrostatins inhibit oxidative lipid damage and cell death in diverse disease models. J Am Chem Soc. 2014;136:4551-6.

26. Mai M, Stefan F, Christoph S, Marcus C, Bornkamm GW, Manfred K. T cell lipid peroxidation induces ferroptosis and prevents immunity to infection. J Exp Med. 2015;212:555-68.

27. Hou B, Zhao Y, Qiang G, Yang X, Xu C, Chen X, et al. Puerarin mitigates diabetic hepatic steatosis and fibrosis by inhibiting TGF$\beta$ signaling pathway activation in type 2 diabetic rats. Oxid Med Cell Longev. 2018;2018:1-13.

28. Yi H, Huang Y, Yang F, Liu W, He S, Hu X. MicroRNA-182 aggravates cerebral ischemia injury by targeting inhibitory member of the ASPP family (iASPP). Arch Biochem Biophysics. 2016;620:S0003986116301412.
29. Ge W, Zhao K, Wang X, Li H, Yu M, He M, et al. iASPP is an antioxidative factor and drives cancer growth and drug resistance by competing with Nrf2 for Keap1 Binding. Cancer Cell. 2017;32:561-73.e566.

30. Silva-Gomes S, Santos AG, Caldas C, Silva CM, Neves JV, Lopes $\mathrm{J}$, et al. Transcription factor NRF2 protects mice against dietary iron-induced liver injury by preventing hepatocytic cell death. J Hepatol. 2014;60:354-61.

31. Bibo K, Xiu-Da S, Yu Z, Haofeng J, Feng G, Shi Y, et al. KEAP1-NRF2 complex in ischemia-induced hepatocellular damage of mouse liver transplants. J Hepatol. 2013;59:1200-7.

32. Zhang LM, Zhang J, Zhang Y, Wang L, Dong L. Interleukin-18 binding protein attenuates lipopolysaccharide-induced acute lung injury in mice via suppression NF- $\mathrm{KB}$ and activation Nrf2 pathway. Biochem Biophys Res Commun. 2018;505:837-42.

33. Liu S, Chen Q, Liu J, Yang X, Zhang Y, Huang F. Sinomenine protects against E.coli-induced acute lung injury in mice through Nrf2-NF-кB pathway. Biomed Pharmacother. 2018;107: 696-702.

34. Yang WS, Sriramaratnam R, Welsch M, Shimada K, Skouta R, Viswanathan V, et al. Regulation of ferroptotic cancer cell death by GPX4. Cell. 2014;156:317-31.

35. Boshuizen M, Van dPK, Von BL, Biemond BJ, Zeerleder SS, Van BR, et al. Therapeutic use of transferrin to modulate anemia and conditions of iron toxicity. Blood Rev. 2017;31: S0268960X17300413.

36. Shi W, Feng B, Xu S, Shen X, Zhang T. Inhibitory effect of compound Chuanxiong Kangxian granules on bleomycin-induced pulmonary fibrosis in rats. Biomed Pharmacother. 2017;96:1179.

37. Kang R, Tang D. Autophagy and ferroptosis-what's the connection? Curr Pathobiol Rep. 2017;5:153. 